\title{
Hamiltonian discontinuous Galerkin FEM for linear, rotating incompressible Euler equations: inertial waves
}

\author{
S. Nurijanyan ${ }^{\mathrm{a}, *}$, J.J.W. van der Vegt ${ }^{\mathrm{a}}$, O. Bokhove $\mathrm{B}^{\mathrm{a}, \mathrm{b}, *}$ \\ ${ }^{a}$ Department of Applied Mathematics, University of Twente, P.O. Box 217, 7500 AE, \\ Enschede, The Netherlands \\ ${ }^{b}$ School of Mathematics, University of Leeds, LS2 9JT, Leeds, U.K.
}

\begin{abstract}
A discontinuous Galerkin finite element method (DGFEM) has been developed and tested for the linear, three-dimensional, rotating incompressible Euler equations. These equations admit complicated wave solutions, which poses numerical challenges.

These challenges concern: (i) discretisation of a divergence-free velocity field; (ii) discretisation of geostrophic boundary conditions combined with nonormal flow at solid walls; (iii) discretisation of the conserved, Hamiltonian dynamics of the inertial-waves; and, (iv) large-scale computational demands owing to the three-dimensional nature of inertial-wave dynamics and possibly its narrow zones of chaotic attraction. These issues have been resolved, for example: (i) by employing Dirac's method of constrained Hamiltonian dynamics to our DGFEM for linear, compressible flows, thus enforcing the incompressibility constraints; (ii) by enforcing no-normal flow at solid walls in a weak form and geostrophic tangential flow along the wall; and, (iii) by applying a symplectic time discretisation.

We compared our simulations with exact solutions of three-dimensional incompressible flows, in (non)rotating periodic and partly periodic cuboids (Poincaré waves). Additional verifications concerned semi-analytical eigenmode solutions in rotating cuboids with solid walls. Finally, a simulation in a
\end{abstract}

\footnotetext{
*Corresponding authors.

Email addresses: s.nurijanyan@math.utwente.nl (S. Nurijanyan), j.j.w.vandervegt@math.utwente.nl (J.J.W. van der Vegt), o.bokhove@math.utwente.nl (O. Bokhove)
} 
tilted rotating tank, yielding more complicated wave dynamics, demonstrates the potential of our new method.

Keywords: Linear Euler equations, Hamiltonian structure, Discontinuous Galerkin method, Inertial waves, Compatible schemes 2000 MSC: 65M60, 65N30, 76B07, 76B15

\section{Introduction}

In the geophysical context, wave motion plays a very important role in energy and angular momentum transport within the oceans and lakes, in particular in the interior of the fluid. These waves often cause mixing, and this mixing forms a very important part of the ocean circulation. Internal gravity (e.g., [42]) and 'gyroscopic' waves, further on referred to as inertial waves (e.g., [I7]), are the main representatives of transverse ocean waves which have their maximum particle displacement not at the free surface, but in the interior of the fluid domain. In contrast to internal gravity waves, where density stratification is the main restoring mechanism, inertial waves exist solely due to the angular momentum stratification. Coriolis forces caused by the rotation of the Earth act as a restoring force on the wave motion. While the influence of rotation in comparison with stratification in geophysical applications is weaker, inertial waves remain of importance in several cases. Inertial waves influence the liquid outer core of the Earth ([23, 22, 3, 34] ), orbiting and/or spinning spaceships and satellites carrying liquid payload ([3, 24]), relatively homogeneous parts of the ocean ([1.9, 4, 12]), lake hydrodynamics ([1]]), and are important in some astrophysical applications ([9]). An important property of these inertial waves is that their propagation direction is determined by ratio of the wave frequency and Coriolis frequency (at twice the rotation rate), and is not altered by the reflection from the boundaries of the fluid domain. The latter results in wave focussing and defocussing phenomena in the absence of a "local reflectional symmetry", in which case the domain walls are asymmetric, i.e., neither parallel nor perpendicular to the rotation axis. Repeated reflection in which wave focusing is dominating gives in general rise to wave attractors: narrow regions onto which the wave energy converges. In a limited set of geometries these attractors were theoretically predicted $([3.3,40,41,[3.5,[22])$ and experimentally observed $([2.5])$, especially in quasi-2D set-ups. The purpose of this work is to provide nu- 
merical tools such that we are able to increase our understanding of inertial waves via numerical simulations of a rotating homogeneous fluid.

Inertial waves are best studied in isolation, in a homogeneous fluid, in the absence of viscosity and nonlinearity. We therefore focus on the development and testing of finite element numerical solution techniques for the linear, three-dimensional incompressible Euler equations in rotating (closed) domains, instead of focussing directly on the more complex Navier-Stokes equations.

It is useful to contrast two types of waves admitted by the linear, incompressible Euler equations: (a) inertial waves in closed rotating domains, and (b) surface-trapped waves in half-closed domains with the free surface of the liquid acting under gravity. Surface waves arise due to the restoring force of gravity at the interface between a heavier fluid (e.g., sea water) and a lighter fluid or vacuum. Linear surface waves in the absence of Coriolis forces only involve the potential-flow component, while the vortical components of the velocity or the vorticity (the three-dimensional curl of the velocity vector) are zero. In contrast, inertial waves involve nonzero vortical components of the velocity and exhibit multi-scale behaviour, especially when wave focusing occurs. These inertial-wave solutions are thus challenging to compute, either analytically or numerically. In addition, the linear three-dimensional Euler equations form a Hamiltonian system. The wave dynamics of both wave types thus concern geometric, Hamiltonian dynamics, as an initial value problem, in which invariants such as mass, energy and phase-space volume derive from this geometric structure. Furthermore, the Hamiltonian system is constrained since the total density is constant and the divergence of the velocity field is zero. Preservation of these discrete invariants in the numerical discretisation ensures numerical stability without any loss of wave amplitude due to artificial numerical damping. The compatible numerical discretisation we aim to develop for these linear incompressible Euler equations should therefore preferably inherit a discrete analog of this characteristic Hamiltonian geometric structure.

To wit, our goal is to develop and test a Hamiltonian discontinuous Galerkin finite element method (DGFEM) for inertial-wave dynamics of the linear, incompressible, three-dimensional, rotating Euler equations. The features of the inertial waves indicate that the following mathematical and numerical challenges should be met: (i) The constraint of incompressibility of the flow, or the zero divergence of the velocity, needs to be inherited by the discretisation in a weak or strong form. This is a classical issue in compu- 
tational fluid dynamics, in which the pressure acts as a Lagrange multiplier to ensure time consistency of the secondary constraint of incompressibility (namely the zero divergence). The zero perturbation density acts here as primary constraint. (ii) The discretisation needs to satisfy the geostrophic balance relations along the wall together with the no-normal flow condition imposed either weakly or strongly. Rotation in combination with the no-normal flow requirement at solid walls yields geostrophic balance conditions on the tangential velocity components. It is nontrivial to satisfy these consistency boundary conditions discretely (e.g., see [प]). (iii) A discrete analog of the geometric Hamiltonian structure needs to be established to ensure conservation properties of the system. In particular, it would guarantee preservation of wave amplitude and phase space volume, such that long-time calculations remain stable and relevant over many wave periods [18]. The use of stable dissipative, time integrators would destroy the carefully preserved geometric structure of the spatial discretisation designed for Hamiltonians in classical mechanics. Hence, symplectic time integrators are required.

The need to deal with local fine scales and the presence of strong gradients led to our choice for discontinuous Galerkin finite element methods in the first place. Furthermore, DGFEM permits large gradients and $h p$-refinement. The computational linear algebra demands are handled by using PETSc [38, 3.9] in our versatile DGFEM software environment hpGEM [32].

The outline of the paper is as follows and concerns all four challenges. In Section 2, we review the equations of motion for the linear compressible and incompressible Euler equations and their Hamiltonian formulations. It also includes an exposition of Dirac's method of constraints for the linear compressible Euler equations, with zero perturbation density as primary constraint [7, 36]. Concerning challenges (i)-(ii), in Section 3, we derive the general Hamiltonian DGFEM for an incompressible flow from the Hamiltonian structure for a compressible flow via Dirac's theory. Concerning challenge (iii), in Section 4, we present a proper time integrator for the presented Hamiltonian dynamics and discuss some of the properties of the resulting time and space discrete numerical schemes. Numerical verifications are given in Section 5, where DGFEM simulations are compared with exact solutions of incompressible flow in a rotating triple-periodic domain and a partially closed cuboid with periodicity in one direction, and with semi-analytical series solutions for incompressible flow in closed cuboids. Additionally, numerical results on chaotic wave attractors are presented. Conclusions are drawn in Section 6. 


\section{Continuum theory for (in)compressible fluid}

\subsection{Governing equations}

Compressible fluid flow in a domain $D$ is governed by the non-linear compressible Euler equations in a rotating frame with angular velocity $\Omega=$ $\left(\Omega_{1}, \Omega_{2}, \Omega_{3}\right)^{T}$ :

$$
\begin{aligned}
& \frac{\partial \hat{\mathbf{u}}}{\partial t}=-\mathbf{2} \Omega \times \hat{\mathbf{u}}-(\hat{\mathbf{u}} \cdot \nabla) \hat{\mathbf{u}}-\hat{\rho}^{-1} \nabla \hat{P}(\hat{\rho}), \\
& \frac{\partial \hat{\rho}}{\partial t}=-\nabla \cdot(\hat{\rho} \hat{\mathbf{u}})
\end{aligned}
$$

where $\hat{\mathbf{u}}=\hat{\mathbf{u}}(x, y, z, t)=(\hat{u}, \hat{v}, \hat{w})^{T}$ is the three-dimensional velocity field, $\hat{\rho}=\hat{\rho}(x, y, z, t)$ a scalar density field, and $\hat{P}=\hat{P}(\hat{\rho})$ the barotropic pressure. Cartesian coordinates $\mathbf{x}=(x, y, z)$ and time $t$ are used; the three-dimensional differential operator is given by $\nabla=(\partial / \partial x, \partial / \partial y, \partial / \partial z)^{T}$. The boundaries of the domain $D$ are denoted by $\partial D=\cup_{i} \partial D_{i}$.

We linearise the compressible Euler equations (四) around a rest state with $\mathbf{u}_{0}=\mathbf{0}$ and $\rho_{0}=$ const., such that $\hat{\mathbf{u}}=\mathbf{0}+\epsilon \mathbf{u}$ and $\hat{\rho}=\rho_{0}+\epsilon \rho$, where $\mathbf{u}$ and $\rho$ are the perturbation velocity and density fields, respectively. Linearisation yields the linear compressible Euler equations in a rotating domain

$$
\begin{aligned}
\frac{\partial \mathbf{u}}{\partial t} & =-\nabla\left(\frac{c_{0}^{2}}{\rho_{0}} \rho\right)-2 \boldsymbol{\Omega} \times \mathbf{u}, \\
\frac{\partial \rho}{\partial t} & =-\nabla \cdot\left(\rho_{0} \mathbf{u}\right)
\end{aligned}
$$

where $c_{0}=\left.\sqrt{\partial \hat{P} / \partial \rho}\right|_{\rho=\rho_{0}}$ is the constant, acoustic wave speed. Two types of boundary conditions will be discussed: periodic and solid-wall boundary conditions. For fixed, solid-wall boundary conditions the normal component of the velocity field at the boundaries is zero $\mathbf{u} \cdot \hat{\mathbf{n}}=0$, with $\hat{\mathbf{n}}$ the outward normal vector at the boundary. If we multiply both sides of the momentum equations ([2a), restricted to the domain boundary, with the normal vector $\hat{\mathbf{n}}$

$$
\frac{\partial(\mathbf{u} \cdot \hat{\mathbf{n}})}{\partial t}=-\nabla\left(\frac{c_{0}^{2}}{\rho_{0}} \rho\right) \cdot \hat{\mathbf{n}}-(2 \boldsymbol{\Omega} \times \mathbf{u}) \cdot \hat{\mathbf{n}}
$$


and apply the no-normal flow condition $\mathbf{u} \cdot \hat{\mathbf{n}}=0$, we obtain a restriction on the density gradient

$$
\frac{c_{0}^{2}}{\rho_{0}} \nabla \rho \cdot \hat{\mathbf{n}}=-(2 \Omega \times \mathbf{u}) \cdot \hat{\mathbf{n}} .
$$

In the absence of domain rotation, the right side of (团) is zero at the boundary, which indicates that the normal component of the density gradient is also zero at the boundary. In contrast, with rotation the normal component of the density gradient is balanced by the projected components of the velocity field. This balance between the density/pressure gradient force and the Coriolis force is called geostrophic balance. Implementation of the boundary condition therefore becomes more challenging due to the mandatory satisfaction of geostrophic balance.

In the limit of zero Mach number, $M_{0}=V_{0} / c_{0} \rightarrow 0$, with $V_{0}$ a reference velocity of the fluid, the linear incompressible Euler equations arise from (घ) as

$$
\begin{aligned}
& \frac{\partial \mathbf{u}}{\partial t}=-2 \Omega \times \mathbf{u}-\nabla P \\
& \nabla \cdot \mathbf{u}=0, \rho=0
\end{aligned}
$$

where $P$ is the pressure. Note that the constraint on the perturbation density $\rho$ ensures that the total density is constant for all time.

\subsection{Hamiltonian framework}

In the following sections we introduce the Hamiltonian framework for linear compressible and incompressible fluid flows, including the connection with the corresponding partial differential equations (PDEs). In general, a Hamiltonian system consists of a phase-space and two geometric objects, an energy functional $\mathcal{H}$ and a Poisson bracket $\{$,$\} [5, 28, 37]. The Hamiltonian$ dynamics is given by the time evolution of a general state functional $\mathcal{F}$ via the bracket form

$$
\frac{d \mathcal{F}}{d t}=\{\mathcal{F}, \mathcal{H}\}
$$

for a specific Hamiltonian functional, or energy, $\mathcal{H}$. This (generalized) Poisson bracket $\{\mathcal{F}, \mathcal{H}\}$ has to satisfy the following properties:

(i) skew-symmetry: $\quad\{\mathcal{F}, \mathcal{H}\}=-\{\mathcal{H}, \mathcal{F}\}$, 
(ii) linearity in the first component: $\{\alpha \mathcal{F}+\beta \mathcal{G}, \mathcal{H}\}=\alpha\{\mathcal{F}, \mathcal{H}\}+\beta\{\mathcal{G}, \mathcal{H}\}$,

(iii) the Jacobi identity: $\{\mathcal{F},\{\mathcal{G}, \mathcal{H}\}\}+\{\mathcal{G},\{\mathcal{H}, \mathcal{F}\}\}+\{\mathcal{H},\{\mathcal{F}, \mathcal{G}\}\}=0$, and:

(iv) the Leibniz identity: $\{\mathcal{F} \mathcal{G}, \mathcal{H}\}=\mathcal{F}\{\mathcal{G}, \mathcal{H}\}+\{\mathcal{F}, \mathcal{H}\} \mathcal{G}$,

where $\alpha$ and $\beta$ are constants, and $\mathcal{F}, \mathcal{G}$ and $\mathcal{H}$ arbitrary functionals. The skew-symmetry of the bracket automatically results in energy conservation: $d \mathcal{H} / d t=\{\mathcal{H}, \mathcal{H}\}=0$.

\subsubsection{Bracket for linearised compressible flow}

Hamiltonian dynamics of compressible fluid flow, cf., [8, [2.9] governed by the linear equations (Z) in $D \subset \mathbb{R}^{3}$ is given by

$$
\frac{d \mathcal{F}}{d t}=\{\mathcal{F}, \mathcal{H}\}=\int_{D}\left(\frac{\delta \mathcal{H}}{\delta \rho} \nabla \cdot \frac{\delta \mathcal{F}}{\delta \mathbf{u}}-\frac{\delta \mathcal{F}}{\delta \rho} \nabla \cdot \frac{\delta \mathcal{H}}{\delta \mathbf{u}}-\frac{\mathbf{2} \Omega}{\rho_{0}} \times \frac{\delta \mathcal{H}}{\delta \mathbf{u}} \cdot \frac{\delta \mathcal{F}}{\delta \mathbf{u}}\right) d \mathbf{x},
$$

with Hamiltonian energy functional

$$
\mathcal{H}=\mathcal{H}[\mathbf{u}, \rho] \equiv \int_{D} \frac{1}{2}\left(\rho_{0} \mathbf{u}^{2}+\frac{c_{0}^{2}}{\rho_{0}} \rho^{2}\right) d \mathbf{x}
$$

The definition of the functional derivative is

$$
\delta \mathcal{H} \equiv \lim _{\epsilon \rightarrow 0} \frac{\mathcal{H}[\mathbf{u}+\epsilon \delta \mathbf{u}, \rho+\epsilon \delta \rho]-\mathcal{H}[\mathbf{u}, \rho]}{\epsilon}=\int_{D}\left[\frac{\delta \mathcal{H}}{\delta \mathbf{u}} \cdot \delta \mathbf{u}+\frac{\delta \mathcal{H}}{\delta \rho} \delta \rho\right] d \mathbf{x} .
$$

The functional derivatives of $\mathcal{H}$ follow from (8) and (9), and are

$$
\frac{\delta \mathcal{H}}{\delta \mathbf{u}}=\rho_{0} \mathbf{u}, \quad \frac{\delta \mathcal{H}}{\delta \rho}=\frac{c_{0}^{2}}{\rho_{0}} \rho .
$$

The Poisson bracket $\{$,$\} in (प) satisfies all properties: skew-symmetry is easy$ to spot from the structure of the bracket; the bracket is obviously bilinear, thus the linearity and Leibniz identity are automatically satisfied; and, the Jacobi identity can be checked directly, given suitable boundary conditions.

To specify Hamiltonian dynamics in the domain $D$ one has to specify appropriate boundary conditions. Mathematical models based on PDEs usually specify boundary conditions on the relevant variables at the boundary. Similarly, in the Hamiltonian formulation boundary conditions can be imposed by choosing appropriate function spaces for the arbitrary functional $\mathcal{F}$. 
As an example, we will show the equivalence between the Hamiltonian

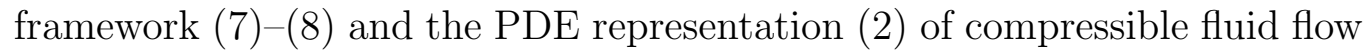
in a rotating domain $D$ bounded by solid walls. The momentum and continuity equations can be obtained if the following functionals are chosen as follows

$$
\begin{aligned}
& \mathcal{F}_{\mathbf{u}} \equiv \int_{D} \mathbf{u}(\mathbf{x}, t) \cdot \mathbf{\Phi}(\mathbf{x}) d \mathbf{x} \\
& \mathcal{F}_{\rho} \equiv \int_{D} \rho(\mathbf{x}, t) \phi(\mathbf{x}) d \mathbf{x},
\end{aligned}
$$

with $\phi \in \mathcal{Q}$ and $\Phi \in \mathcal{Y}$ arbitrary test functions, where

$$
\begin{aligned}
& \mathcal{Q}=\left\{\phi \in L^{2}(D)\right\} \\
& \mathcal{Y}=\left\{\boldsymbol{\Phi} \in\left(L^{2}(D)\right)^{3} \text { and } \nabla \cdot \boldsymbol{\Phi} \in L^{2}(D): \hat{\mathbf{n}} \cdot \boldsymbol{\Phi}=0 \text { at } \partial D\right\},
\end{aligned}
$$

and $L^{2}(D)$ is the space of square integrable functions on $D$. To incorporate slip flow boundary conditions at $\partial D$ we restrict the space for the test functions $\boldsymbol{\Phi}$ at the boundary. Corresponding functional derivatives of (एa) and (116) thus become

$$
\frac{\delta \mathcal{F}_{\rho}}{\delta \rho}=\phi(\mathbf{x}) \text { and } \frac{\delta \mathcal{F}_{\mathbf{u}}}{\delta \mathbf{u}}=\boldsymbol{\Phi}(\mathbf{x}) \text {, with } \frac{\delta \mathcal{F}_{\mathbf{u}}}{\delta \mathbf{u}} \cdot \hat{\mathbf{n}}=0 \text { at } \partial D .
$$

Using functionals (एa) and (एש) , with corresponding functional derivatives

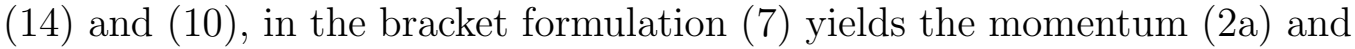
continuity (2D) equations for linearised compressible flow, respectively. We also used Gauss' law combined with ([4]). The restricted test function arising from functional $\mathcal{F}_{\mathbf{u}}$ ensures the satisfaction of the boundary conditions at the PDE level.

\subsubsection{Construction of a Dirac-bracket for linearised incompressible flow}

Dirac's theory of constrained Hamiltonian systems ([10, 36, 43:]) is used to derive the linearised incompressible Euler equations as the limit of the Hamiltonian structure of the linearised compressible Euler equations. Basically, Dirac's theory enforces a constant density constraint via Lagrange multipliers onto the derived compressible Hamiltonian framework $[\mathbf{z}, \mathbf{8}]$.

Due to linearisation, the constant total density constraint $\hat{\rho}=$ const transforms into the perturbation density constraint

$$
\rho(\mathbf{x})=0 .
$$


It will act as a primary constraint, to be incorporated into the compressible Hamiltonian dynamics (U) via a Lagrange multiplier field. In a consistent theory, the constraint must be preserved by the evolution of the system. This leads to several possible outcomes: (i) the consistency requirement results into, modulo constraints, an equation of essentially the form $1=0$; (ii) it leads to an equation of the form $0=0$; (iii) we obtain an equation which resolves the unknown Lagrange multiplier, or (iv) it yields a secondary constraint. Case (i) implies inconsistent equations of motion; they do not posses any solution. Case (ii) is the desired outcome. Case (iv) introduces new secondary constraints, preservation of which must be checked by repeating the procedure until either we encounter case (i) or all constraints lead to case (ii). This is the main idea of Dirac's algorithm.

A Lagrange multiplier $\lambda_{\rho}(\mathbf{x}, t)$ is introduced to enforce the primary constraint. This constraint, or any arbitrary functional $\mathcal{F}[\rho]$ thereof, must be preserved in time. Hence, the evolution of such a functional must remain naught, i.e.,

$$
\frac{d \mathcal{F}[\rho]}{d t}=0=\{\mathcal{F}[\rho], \mathcal{H}\}+\int_{D} \lambda_{\rho}\left(\mathbf{x}^{\prime}\right)\left\{\mathcal{F}[\rho], \rho\left(\mathbf{x}^{\prime}\right)\right\} d \mathbf{x}^{\prime}
$$

From Poisson bracket $(\mathbb{\square})$, we deduce that $\left\{\mathcal{F}[\rho], \rho\left(\mathbf{x}^{\prime}\right)\right\}=0$ and, therefore, the Lagrange multiplier remains undetermined. It gives, however, rise to a secondary constraint

$$
0=\{\mathcal{F}[\rho], \mathcal{H}\}=-\int_{D} \frac{\delta \mathcal{F}[\rho]}{\delta \rho} \nabla \cdot\left(\rho_{0} \mathbf{u}(\mathbf{x})\right) d \mathbf{x}
$$

Since the functional $\mathcal{F}[\rho]$ is arbitrary in (ㅍ) , it follows that

$$
\nabla \cdot \mathbf{u}=0
$$

should hold as well. Note that $\delta \mathcal{F}[\rho] / \delta \rho$ serves as arbitrary test function and that the secondary constraint implies that the velocity is divergence-free. Next, both constraints

$$
\rho(\mathbf{x})=0 \quad \text { and } \quad \Delta(\mathbf{x})=\nabla \cdot \mathbf{u}(\mathbf{x})=0
$$

will be enforced simultaneously as primary constraints, also in time.

For this reason, we introduce Lagrange multipliers $\lambda_{\rho}=\lambda_{\rho}(\mathbf{x}, t)$ and $\lambda_{\Delta}=\lambda_{\Delta}(\mathbf{x}, t)$. The two consistency requirements are stated in weak form 
by using two (different) arbitrary functionals $\mathcal{F}[\rho]$ and $\mathcal{F}[\Delta]$, as follows

$$
\begin{aligned}
\frac{d \mathcal{F}[\rho]}{d t}=0=\{\mathcal{F}[\rho], \mathcal{H}\} & +\int_{D} \lambda_{\Delta}\left(\mathbf{x}^{\prime}\right)\left\{\mathcal{F}[\rho], \Delta\left(\mathbf{x}^{\prime}\right)\right\} d \mathbf{x}^{\prime} \\
\frac{d \mathcal{F}[\Delta]}{d t}=0=\{\mathcal{F}[\Delta], \mathcal{H}\} & +\int_{D} \lambda_{\rho}\left(\mathbf{x}^{\prime}\right)\left\{\mathcal{F}[\Delta], \rho\left(\mathbf{x}^{\prime}\right)\right\} d \mathbf{x}^{\prime} \\
& +\int_{D} \lambda_{\Delta}\left(\mathbf{x}^{\prime}\right)\left\{\mathcal{F}[\Delta], \Delta\left(\mathbf{x}^{\prime}\right)\right\} d \mathbf{x}^{\prime},
\end{aligned}
$$

where we omitted stating the explicit time dependence. An elaborate calculation of the brackets in (20a) yields

$$
0=\int_{D} \frac{\delta \mathcal{F}}{\delta \rho}\left(-\nabla \cdot\left(\rho_{0} \mathbf{u}\right)+\nabla^{2} \lambda_{\Delta}\right) d \mathbf{x}-\int_{\partial D} \frac{\delta \mathcal{F}}{\delta \rho} \hat{\mathbf{n}} \cdot \nabla \lambda_{\Delta} d S
$$

with surface element $d S$. By using the secondary constraint in (20), and the arbitrariness of the functional $\mathcal{F}[\rho]$ in the interior and at the boundary, we find that

$$
\nabla^{2} \lambda_{\Delta}=0 \quad \text { with } \quad \hat{\mathbf{n}} \cdot \nabla \lambda_{\Delta}=0 .
$$

Its solution is $\lambda_{\Delta}=$ cst.

To analyse $(20 \mathrm{~b})$, we first relate the functional derivative of $\mathcal{F}[\Delta]$ with respect to $\Delta$ to the one with respect to $\mathbf{u}$, as follows

$$
\delta \mathcal{F}[\Delta]=\int_{D} \frac{\delta \mathcal{F}[\Delta]}{\delta \Delta} \delta \Delta d \mathbf{x}=-\int_{D} \nabla \frac{\delta \mathcal{F}[\Delta]}{\delta \Delta} \cdot \delta \mathbf{u} d \mathbf{x}
$$

where we used that $\hat{\mathbf{n}} \cdot \delta \mathbf{u}=0$. The last term in (20b) cancels after an integration by parts, by using the additional boundary conditions $\hat{\mathbf{n}} \cdot \nabla \lambda_{\Delta}=0$ and $\hat{\mathbf{n}} \cdot \nabla(\delta \mathcal{F}[\Delta] / \delta \Delta)=0$ at $\partial D$. We subsequently find that $(20 \mathrm{~b})$ becomes

$$
0=\int_{D} \frac{\delta \mathcal{F}}{\delta \Delta}\left(\nabla \cdot(2 \boldsymbol{\Omega} \times \mathbf{u})+\nabla^{2} \lambda_{\rho}\right) d \mathbf{x}-\int_{\partial D} \frac{\delta \mathcal{F}}{\delta \Delta} \hat{\mathbf{n}} \cdot\left(2 \boldsymbol{\Omega} \times \mathbf{u}+\nabla \lambda_{\rho}\right) d S .
$$

The arbitrariness of $\mathcal{F}[\Delta]$ in $(\mathbb{Z}])$, in the interior and at the boundary, then implies that

$$
\nabla \cdot(2 \boldsymbol{\Omega} \times \mathbf{u})+\nabla^{2} \lambda_{\rho}=0 \quad \text { on } D \quad \text { with } \quad\left(2 \boldsymbol{\Omega} \times \mathbf{u}+\nabla \lambda_{\rho}\right) \cdot \hat{\mathbf{n}}=0 \quad \text { on } \partial D .
$$

Details in the above calculations have been relegated to Appendix .1. 
The bracket formulation for incompressible flow is now given by

$$
\frac{d \mathcal{F}[\mathbf{u}]}{d t}=\{\mathcal{F}, \mathcal{H}\}+\int_{D} \lambda_{\rho}\left(\mathbf{x}^{\prime}\right)\left\{\mathcal{F}, \rho\left(\mathbf{x}^{\prime}\right)\right\} d \mathbf{x}^{\prime}
$$

The dynamics is then obtained from (26) combined with (24) for the Lagrange multiplier $\left(\lambda=\lambda_{\rho}\right)$

$$
\begin{aligned}
\frac{d \mathcal{F}}{d t} & =\{\mathcal{F}, \mathcal{H}\}_{\text {inc }} \equiv \int_{D}\left[-\frac{2 \Omega}{\rho_{0}} \times \frac{\delta \mathcal{H}}{\delta \mathbf{u}(\mathbf{x})} \cdot \frac{\delta \mathcal{F}}{\delta \mathbf{u}(\mathbf{x})}+\lambda(\mathbf{x}) \nabla \cdot \frac{\delta \mathcal{F}}{\delta \mathbf{u}(\mathbf{x})}\right] d \mathbf{x}, \\
0 & =\int_{D} \frac{\delta \mathcal{F}}{\delta \Delta}\left(\nabla \cdot(2 \Omega \times \mathbf{u})+\nabla^{2} \lambda\right) d \mathbf{x}-\int_{\partial D}(2 \Omega \times \mathbf{u}+\nabla \lambda) \cdot \hat{\mathbf{n}} \frac{\delta \mathcal{F}}{\delta \Delta} d S,
\end{aligned}
$$

with constrained energy functional

$$
\mathcal{H}=\int_{D} \frac{1}{2} \rho_{0} \mathbf{u}^{2} d \mathbf{x}
$$

It is obtained after application of the primary constraint $\rho=0$. The incompressible, linear Euler equations can be derived from (27) by choosing functionals

$$
\mathcal{F}_{\mathbf{u}} \equiv \int_{D} \mathbf{u}(\mathbf{x}, t) \cdot \mathbf{\Phi}(\mathbf{x}) d \mathbf{x} \quad \text { and } \quad \mathcal{F}_{\Delta} \equiv \int_{D} \Delta(\mathbf{x}, t) \widetilde{\phi}(\mathbf{x}) d \mathbf{x}
$$

where $\mathbf{\Phi}(\mathbf{x}) \in \mathcal{Y}$ and $\widetilde{\phi}(\mathbf{x}) \in \mathcal{Q}$ with the additional requirement that $\hat{\mathbf{n}} \cdot \nabla \widetilde{\phi}=$ 0 . The functionals in ( 28$)$ lead to the system of equations

$$
\frac{\partial \mathbf{u}}{\partial t}=-\nabla \lambda-2 \boldsymbol{\Omega} \times \mathbf{u} \text { and } \nabla^{2} \lambda=-\nabla \cdot(2 \boldsymbol{\Omega} \times \mathbf{u}),
$$

with $\operatorname{slip}$ flow $\mathbf{u} \cdot \hat{\mathbf{n}}=0$ and geostrophic balance $(2 \Omega \times \mathbf{u}+\nabla \lambda) \cdot \hat{\mathbf{n}}=0$ at the solid-wall boundary. Notice that the Lagrange multiplier $\lambda=P$ plays the role of the pressure $P$.

\section{Discrete Hamiltonian formulation}

Discretisations of the earlier derived compressible and incompressible continuous Hamiltonian formulations will be derived next. There are two possible choices for a derivation of discrete Hamiltonian dynamics for incompressible fluid flow: direct discretisation of the continuous bracket formulation ([27) for incompressible fluid flow, or application of Dirac's theory on 
the discretised Hamiltonian formulation of compressible flow. The latter approach is preferable for several reasons: (i) a discretisation of the compressible Hamiltonian formulation is becoming an intermediate check point for the introduced discretisation algorithm; (ii) avoidance of dealing with discontinuities of unknown Lagrange multipliers simplifies the process; and, (iii) the relatively easy incorporation of boundary conditions which are set automatically by Dirac's theory given the proper boundary conditions for the compressible case. Before proceeding to a discontinuous Galerkin FEM discretisation, we demonstrate key aspects of the algorithm on a finite volume (FV) discretisation of the compressible Hamiltonian formulation with consecutive application of Dirac's theory on a discrete level. This FV discretisation is equivalent to a DG discretisation with constant basis functions.

\subsection{Finite volume discretisation for linear Euler equations}

\subsubsection{Discrete compressible dynamics}

The three-dimensional linear compressible Euler equations (घ) are considered in a periodic rectangular parallelepiped, where an equidistant mesh is introduced. The equations are scaled for simplicity such that we effectively can take $\rho_{0}=c_{0}=1$ in ( $(2)$ (hereafter). A tessellation of this triple periodic domain results in a collection of elements $K$ with $(i, j, k)$ index numbering. A $\mathrm{FV}$ discretisation for the scaled version of compressible Euler equations (च), with a chosen "antisymmetric $\theta$ scheme" for the spatial derivatives, yields the following discrete equations

$$
\left.d t\left(\begin{array}{c}
\bar{U}_{i, j, k} \\
\bar{V}_{i, j, k} \\
\bar{W}_{i, j, k} \\
\bar{R}_{i, j, k}
\end{array}\right)=-\left(\begin{array}{c}
\bar{U}_{i, j, k} \\
\bar{V}_{i, j, k} \\
\bar{W}_{i, j, k}
\end{array}\right)\right)-\left(\begin{array}{c}
\bar{G}_{i, j, k}^{1} \\
\bar{G}_{i, j, k}^{2} \\
\bar{G}_{i, j, k}^{3} \\
\bar{G}_{i, j, k}^{4}
\end{array}\right),
$$

where $\bar{U}_{i, j, k}=\bar{U}_{i, j, k}(t), \bar{V}_{i, j, k}=\bar{V}_{i, j, k}(t), \bar{W}_{i, j, k}=\bar{W}_{i, j, k}(t)$ and $\bar{R}_{i, j, k}=\bar{R}_{i, j, k}(t)$ are the time-dependent mean values of $u, v, w, \rho$ in the $(i, j, k)$-th element 
and the flux functions are defined by

$$
\begin{aligned}
\bar{G}_{i, j, k}^{1}= & \frac{\left(\bar{R}_{i+1, j, k}(1-\theta)+\bar{R}_{i, j, k} \theta\right)-\left(\bar{R}_{i, j, k}(1-\theta)+\bar{R}_{i-1, j, k} \theta\right)}{\Delta x}, \\
\bar{G}_{i, j, k}^{2}=- & \frac{\left(\bar{R}_{i, j+1, k}(1-\theta)+\bar{R}_{i, j, k} \theta\right)-\left(\bar{R}_{i, j, k}(1-\theta)+\bar{R}_{i, j-1, k} \theta\right)}{\Delta y}, \\
\bar{G}_{i, j, k}^{3}=- & \frac{\left(\bar{R}_{i, j, k+1}(1-\theta)+\bar{R}_{i, j, k} \theta\right)-\left(\bar{R}_{i, j, k}(1-\theta)+\bar{R}_{i, j, k-1} \theta\right)}{\Delta z}, \\
\bar{G}_{i, j, k}^{4}=- & \frac{\left(\bar{U}_{i, j, k}(1-\theta)+\bar{U}_{i+1, j, k} \theta\right)-\left(\bar{U}_{i-1, j, k}(1-\theta)+\bar{U}_{i, j, k} \theta\right)}{\Delta x} \\
& -\frac{\left(\bar{V}_{i, j, k}(1-\theta)+\bar{V}_{i, j+1, k} \theta\right)-\left(\bar{V}_{i, j-1, k}(1-\theta)+\bar{V}_{i, j, k} \theta\right)}{\Delta y} \\
& -\frac{\left(\bar{W}_{i, j, k}(1-\theta)+\bar{W}_{i, j, k+1} \theta\right)-\left(\bar{W}_{i, j, k-1}(1-\theta)+\bar{W}_{i, j, k} \theta\right)}{\Delta z},
\end{aligned}
$$

with $\Delta x, \Delta y$, and $\Delta z$ the respective mesh sizes, and $0 \leq \theta \leq 1$.

Energy conservation can be shown by a series of straightforward calculations: multiply equations (茴) by $\left(\bar{U}_{(i, j, k)}, \bar{V}_{(i, j, k)}, \bar{W}_{(i, j, k)}, \bar{R}_{(i, j, k)}\right)$, and sum over all elements. Discretisation (30) then leads to energy conservation, i.e.,

$$
\frac{d H}{d t}=0, \quad \text { with } \quad H=\sum_{(i, j, k)} \frac{1}{2}\left(\bar{U}_{i, j, k}^{2}+\bar{V}_{i, j, k}^{2}+\bar{W}_{i, j, k}^{2}+\bar{R}_{i, j, k}^{2}\right) .
$$

The latter result suggests there is a discrete Hamiltonian formulation for (30). We first calculate the partial derivatives of the Hamiltonian

$$
\frac{\partial H}{\partial \bar{U}_{i, j, k}}=\bar{U}_{i, j, k}, \quad \frac{\partial H}{\partial \bar{V}_{i, j, k}}=\bar{V}_{i, j, k}, \quad \frac{\partial H}{\partial \bar{W}_{i, j, k}}=\bar{W}_{i, j, k}, \quad \frac{\partial H}{\partial \bar{R}_{i, j, k}}=\bar{R}_{i, j, k}
$$

and use these in the right hand side of (30) . Subsequently, we multiply the four equations in (B)a by $\partial F / \partial \bar{U}_{i, j, k}, \partial F / \partial \bar{V}_{i, j, k}, \partial F / \partial \bar{W}_{i, j, k}$, and $\partial F / \partial \bar{R}_{i, j, k}$, respectively, add them up, and sum over all cells. After some algebraic 
manipulations, it yields the Hamiltonian finite-dimensional dynamics

$$
\begin{aligned}
& \frac{d F}{d t}=[F, H]_{c_{\theta}} \\
& \equiv-\frac{\partial F}{\partial \overline{\mathbf{U}}_{\mathbf{k}}} \cdot 2 \boldsymbol{\Omega} \times \frac{\partial H}{\partial \overline{\mathbf{U}}_{\mathbf{k}}} \\
& +\frac{\partial H}{\partial \overline{\mathbf{U}}_{\mathbf{k}}} \cdot\left((1-\theta)\left(\begin{array}{c}
\frac{1}{\Delta x}\left(\frac{\partial F}{\partial \bar{R}_{i+1}}-\frac{\partial F}{\partial \bar{R}_{\mathbf{k}}}\right) \\
\frac{1}{\Delta y}\left(\frac{\partial F}{\partial \bar{R}_{j+1}}-\frac{\partial F}{\partial \bar{R}_{\mathbf{k}}}\right) \\
\frac{1}{\Delta z}\left(\frac{\partial F}{\partial \bar{R}_{k+1}}-\frac{\partial F}{\partial \bar{R}_{\mathbf{k}}}\right)
\end{array}\right)+\theta\left(\begin{array}{c}
\frac{1}{\Delta x}\left(\frac{\partial F}{\partial \bar{R}_{\mathbf{k}}}-\frac{\partial F}{\partial \bar{R}_{i-1}}\right) \\
\frac{1}{\Delta y}\left(\frac{\partial F}{\partial \bar{R}_{\mathbf{k}}}-\frac{\partial F}{\partial \bar{R}_{j-1}}\right) \\
\frac{1}{\Delta z}\left(\frac{\partial F}{\partial \bar{R}_{\mathbf{k}}}-\frac{\partial F}{\partial \bar{R}_{k-1}}\right)
\end{array}\right)\right) \\
& -\frac{\partial F}{\partial \overline{\mathbf{U}}_{\mathbf{k}}} \cdot\left((1-\theta)\left(\begin{array}{c}
\frac{1}{\Delta x}\left(\frac{\partial H}{\partial \bar{R}_{i+1}}-\frac{\partial H}{\partial \bar{R}_{\mathbf{k}}}\right) \\
\frac{1}{\Delta y}\left(\frac{\partial H}{\partial \bar{R}_{j+1}}-\frac{\partial H}{\partial \bar{R}_{\mathbf{k}}}\right) \\
\frac{1}{\Delta z}\left(\frac{\partial H}{\partial \bar{R}_{k+1}}-\frac{\partial H}{\partial \bar{R}_{\mathbf{k}}}\right)
\end{array}\right)+\theta\left(\begin{array}{c}
\frac{1}{\Delta x}\left(\frac{\partial H}{\partial \bar{R}_{\mathbf{k}}}-\frac{\partial H}{\partial \bar{R}_{i-1}}\right) \\
\frac{1}{\Delta y}\left(\frac{\partial H}{\partial \bar{R}_{\mathbf{k}}}-\frac{\partial H}{\partial \bar{R}_{i-1}}\right) \\
\frac{1}{\Delta z}\left(\frac{\partial H}{\partial \bar{R}_{\mathbf{k}}}-\frac{\partial H}{\partial \bar{R}_{k-1}}\right)
\end{array}\right)\right),
\end{aligned}
$$

where $\overline{\mathbf{U}}=(\bar{U}, \bar{V}, \bar{W})^{T}$ and $\mathbf{k}=(i, j, k)$, and we used the shorthand notation $R_{i+1}=R_{i+1, j, k}$, et cetera. Repeated indices indicate summation, if not stated otherwise. By inspection, (B3) is seen to be anti-symmetric, and independent of the variables involved. It therefore satisfies all requirement of a (noncanonical) Hamiltonian system. The $c_{\theta}$ subscript indicates the cosymplectic form of the Poisson bracket for a FV discretisation of the compressible Euler equations. Since several brackets appear below, we will use such subscripts to distinguish the different brackets used hereafter.

\subsubsection{Discrete incompressible dynamics}

The discrete compressible Hamiltonian dynamics (B:3) with (B]) is our starting point for Dirac's theory of constraints. It will be used to enforce the density as a constraint into the compressible Hamiltonian formulation. For simplicity of illustration, we will only consider the case with $\theta=0$ in (B3]). 
With some minor index renumbering, we obtain

$$
\begin{aligned}
\frac{d F}{d t} & =[F, H]_{c_{0}} \equiv-\left(2 \Omega \times \frac{\partial H}{\partial \overline{\mathbf{U}}_{\mathbf{k}}}\right) \cdot \frac{\partial F}{\partial \overline{\mathbf{U}}_{\mathbf{k}}} \\
& -\left(\frac{\partial H}{\partial \bar{R}_{i+1, j, k}}-\frac{\partial H}{\partial \bar{R}_{\mathbf{k}}}\right) \frac{1}{\Delta x} \frac{\partial F}{\partial \bar{U}_{\mathbf{k}}}-\left(\frac{\partial H}{\partial \bar{R}_{i, j+1, k}}-\frac{\partial H}{\partial \bar{R}_{\mathbf{k}}}\right) \frac{1}{\Delta y} \frac{\partial F}{\partial \bar{V}_{\mathbf{k}}} \\
& -\left(\frac{\partial H}{\partial \bar{R}_{i, j, k+1}}-\frac{\partial H}{\partial \bar{R}_{\mathbf{k}}}\right) \frac{1}{\Delta z} \frac{\partial F}{\partial \bar{W}_{\mathbf{k}}}-\left(\frac{\partial H}{\partial \bar{U}_{\mathbf{k}}}-\frac{\partial H}{\partial \bar{U}_{i-1, j, k}}\right) \frac{1}{\Delta x} \frac{\partial F}{\partial \bar{R}_{\mathbf{k}}} \\
& -\left(\frac{\partial H}{\partial \bar{V}_{\mathbf{k}}}-\frac{\partial H}{\partial \bar{V}_{i, j-1, k}}\right) \frac{1}{\Delta y} \frac{\partial F}{\partial \bar{R}_{\mathbf{k}}}-\left(\frac{\partial H}{\partial \bar{W}_{\mathbf{k}}}-\frac{\partial H}{\partial \bar{W}_{i, j, k-1}}\right) \frac{1}{\Delta z} \frac{\partial F}{\partial \bar{R}_{\mathbf{k}}}
\end{aligned}
$$

For the continuous problem the primary constraint is the zero perturbation density, $\rho(x, y, z, t)=0$. For the FV discretisation, it means that the mean value of the density is zero everyhwere,

$$
\bar{R}_{\mathrm{k}}=0 .
$$

The primary constraints should hold in time, as a consistency requirement,

$$
0=\frac{d \bar{R}_{\mathbf{k}}}{d t}=\left[\bar{R}_{\mathbf{k}}, H\right]_{c_{0}}+\mu_{\mathbf{p}}\left[\bar{R}_{\mathbf{k}}, \bar{R}_{\mathbf{p}}\right]_{c_{0}}
$$

with Lagrange multipliers $\mu_{\mathbf{p}}$ and $\mathbf{p}=(p, q, r)$. From (34), it follows that $\left[R_{\mathbf{k}}, R_{\mathbf{p}}\right]_{c_{0}}=0$. The Lagrange multiplier thus remains undetermined and a secondary constraint arises from (36) as

$$
\begin{aligned}
E_{\mathbf{k}} \equiv & -\left[\bar{R}_{\mathbf{k}}, H\right]_{c_{0}}=\left(\frac{\partial H}{\partial \bar{U}_{\mathbf{k}}}+\frac{\partial H}{\partial \bar{U}_{i-1, j, k}}\right) \frac{1}{\Delta x} \\
& \left(\frac{\partial H}{\partial \bar{V}_{\mathbf{k}}}+\frac{\partial H}{\partial \bar{V}_{i, j-1, k}}\right) \frac{1}{\Delta y}+\left(\frac{\partial H}{\partial \bar{W}_{\mathbf{k}}}+\frac{\partial H}{\partial \bar{W}_{i, j, k-1}}\right) \frac{1}{\Delta z} \\
= & \frac{\left(\bar{U}_{\mathbf{k}}-\bar{U}_{i-1, j, k}\right)}{\Delta x}+\frac{\left(\bar{V}_{\mathbf{k}}-\bar{V}_{i, j-1, k}\right)}{\Delta y}+\frac{\left(\bar{W}_{\mathbf{k}}-\bar{W}_{i, j, k-1}\right)}{\Delta z}=0 .
\end{aligned}
$$

A closer look at the constraint (B7) shows that it is a first-order discretization of the divergence-free velocity condition (ए8). Subsequently, both constraints 
are enforced together, with mandatory preservation in time

$$
\begin{aligned}
& 0=\frac{d \bar{R}_{\mathbf{k}}}{d t}=\left[\bar{R}_{\mathbf{k}}, H\right]_{c_{0}}+\lambda_{\mathbf{p}}^{U}\left[\bar{R}_{\mathbf{k}}, E_{\mathbf{p}}\right]_{c_{0}}, \\
& 0=\frac{d E_{\mathbf{k}}}{d t}=\left[E_{\mathbf{k}}, H\right]_{c_{0}}+\lambda_{\mathbf{p}}^{R}\left[E_{\mathbf{k}}, \bar{R}_{\mathbf{p}}\right]_{c_{0}}+\lambda_{\mathbf{p}}^{U}\left[E_{\mathbf{k}}, E_{\mathbf{p}}\right]_{c_{0}} .
\end{aligned}
$$

Application of primary constraint (B5) in (38a) yields $\lambda_{\mathbf{p}}^{U}\left[\bar{R}_{\mathbf{k}}, E_{\mathbf{p}}\right]_{c_{0}}=0$. The latter equation is a FV discretisation of the Laplacian, as follows shortly, and has as solution $\lambda_{\mathbf{p}}^{U}=0$. That result simplifies (38b) to

$$
0=\left[E_{\mathbf{k}}, H\right]_{c_{0}}+\lambda_{\mathbf{p}}^{R}\left[E_{\mathbf{k}}, \bar{R}_{\mathbf{p}}\right]_{c_{0}} .
$$

Further valuation of (B.9) using (B3) gives an equation for $\lambda \equiv \lambda^{R}$

$$
\begin{aligned}
& \frac{\lambda_{i+1, j, k}-2 \lambda_{\mathbf{k}}+\lambda_{i-1, j, k}}{\Delta x^{2}}+\frac{\lambda_{i, j+1, k}-2 \lambda_{\mathbf{k}}+\lambda_{i, j-1, k}}{\Delta y^{2}} \\
& +\frac{\lambda_{i, j, k+1}-2 \lambda_{\mathbf{k}}+\lambda_{i, j, k-1}}{\Delta z^{2}}=-\frac{\left[2 \Omega \times\left(\frac{\partial H}{\partial \overline{\mathbf{U}}_{\mathbf{k}}}-\frac{\partial H}{\partial \overline{\mathbf{U}}_{i-1, j, k}}\right)\right]_{1}}{\Delta x} \\
& -\frac{\left[2 \Omega \times\left(\frac{\partial H}{\partial \overline{\mathbf{U}}_{\mathbf{k}}}-\frac{\partial H}{\partial \overline{\mathbf{U}}_{i, j-1, k}}\right)\right]_{2}}{\Delta y}-\frac{\left[2 \Omega \times\left(\frac{\partial H}{\partial \overline{\mathbf{U}}_{\mathbf{k}}}-\frac{\partial H}{\partial \overline{\mathbf{U}}_{i, j, k-1}}\right)\right]_{3}}{\Delta z} .
\end{aligned}
$$

Note that (40) is a discretisation of the Laplacian acting on the Lagrange multiplier, $\nabla^{2} \lambda$, on the left-hand-side and the divergence of the rotational effects on the right-hand-side, corresponding to a discrete version of the continuous case (25). Finally, the bracket for the incompressible case becomes

$$
\begin{aligned}
\frac{d F}{d t}=[F, H]_{c_{0}}^{i n c} & \equiv-\left(2 \Omega \times \frac{\partial H}{\partial \overline{\mathbf{U}}_{\mathbf{k}}}\right) \cdot \frac{\partial F}{\partial \overline{\mathbf{U}}_{\mathbf{k}}}-\frac{\lambda_{i+1, j, k}-\lambda_{\mathbf{k}}}{\Delta x} \frac{\partial F}{\partial \bar{U}_{\mathbf{k}}} \\
& -\frac{\lambda_{i, j+1, k}-\lambda_{i, j, k}}{\Delta y} \frac{\partial F}{\partial \bar{V}_{\mathbf{k}}}-\frac{\lambda_{i, j, k+1}^{U}-\lambda_{\mathbf{k}}^{U}}{\Delta z} \frac{\partial F}{\partial \bar{W}_{\mathbf{k}}} .
\end{aligned}
$$

Hence, the constrained dynamics for incompressible fluid flow results in the Dirac-bracket formulation (40) coupled with (40) for the Lagrange multiplier $\lambda$, and the discrete energy functional

$$
H=\sum_{(i, j, k)} \frac{1}{2}\left(\bar{U}_{\mathbf{k}}^{2}+\bar{V}_{\mathbf{k}}^{2}+\bar{W}_{\mathbf{k}}^{2}\right) .
$$


Proofs of energy conservation and preservation of zero divergence in time will be presented later for the more general, DGFEM discretisation of incompressible Hamiltonian dynamics.

\subsection{Discontinuous Galerkin FEM discretisation for the linearised Euler equa- tions}

In this section, we will introduce a discontinuous Galerkin FEM discretisation that preserves the Hamiltonian structure of linear, compressible and incompressible flows. The FV discretisation of the Hamiltonian system presented above is used as a guide in the choice of the numerical flux.

\subsubsection{Finite element space}

Let $\mathcal{I}_{h}$ denote a tessellation of the domain $D$ with elements $K$. The set of all edges in the tessellation $\mathcal{I}_{h}$ is $\Gamma$, with $\Gamma_{i}$ the set of interior edges and $\Gamma_{D}$ the set of edges at the domain boundary $\partial D$. Additional notation is introduced for the numerical flux, to be introduced shortly. Let $e$ be a face between "left" and "right" elements $K_{L}$ and $K_{R}$, respectively, with corresponding outward normals $\mathbf{n}_{L}$ and $\mathbf{n}_{R}$. When $f$ is a continuous function on $K_{L}$ and $K_{R}$, but possibly discontinuous across the face $e$, let $f_{L}=\left.\left(\left.f\right|_{K_{L}}\right)\right|_{e}$ and $f_{R}=\left.\left(\left.f\right|_{K_{R}}\right)\right|_{e}$ denote the left and right traces, respectively. Let $\mathcal{P}^{p}(K)$ be the space of polynomials of at most degree $p$ on $K \in \mathcal{I}_{h}$, with $p \geq 0$. The finite element spaces $\mathcal{Q}_{h}$ and $\mathcal{Y}_{h}$ required are

$$
\begin{aligned}
& \mathcal{Q}_{h}=\left\{q \in L^{2}(D):\left.q\right|_{K} \in \mathcal{P}^{p}(K), \forall K \in \mathcal{I}_{h}\right\}, \\
& \mathcal{Y}_{h}=\left\{\boldsymbol{Y} \in\left(L^{2}(D)\right)^{3}:\left.\boldsymbol{Y}\right|_{K} \in\left(\mathcal{P}^{p}(K)\right)^{3}, \forall K \in \mathcal{I}_{h}\right\} .
\end{aligned}
$$

The number of degrees of freedom on an element is denoted by $N_{K}=$ $\operatorname{dim}\left(\mathcal{P}^{p}(K)\right)$.

The discrete energy on the tessellated domain, cf. ( $\mathbb{8})$, thus becomes

$$
H=\frac{1}{2} \sum_{K} \int_{K}\left(\mathbf{u}_{h}^{2}+\rho_{h}^{2}\right) d K,
$$

where $\rho_{h} \in \mathcal{Q}_{h}$ and $\mathbf{u}_{h} \in \mathcal{Y}_{h}$. Corresponding variational derivatives are

$$
\frac{\delta H}{\delta \mathbf{u}_{\mathbf{h}}}=\mathbf{u}_{h} \quad \text { and } \quad \frac{\delta H}{\delta \rho_{h}}=\rho_{h} .
$$

There is some abuse of notation here, because we use functions $F$ and $H$ for functionals. However, if approximations $\mathbf{u}_{h}$ and $\rho_{h}$ are viewed as finitedimensional expansions, then function derivatives with respect to the expansion coefficients emerge. 


\subsubsection{Hamiltonian DGFEM discretisation for linearised compressible flow}

In this section, we derive a DGFEM discretisation of the Hamiltonian structure for linearised compressible flow (ש). The specific functional $F\left[\mathbf{u}_{h}\right] \equiv$ $\int_{D} \mathbf{u}_{h} \cdot \boldsymbol{\Phi} d \mathbf{x}$ is chosen to obtain the discretised momentum equations in a Hamiltonian framework, with $\boldsymbol{\Phi} \in \mathcal{Y}_{h}$ an arbitrary test function. The functional derivative with respect to the velocity thus equals

$$
\frac{\delta F}{\delta \mathbf{u}_{h}}=\boldsymbol{\Phi}
$$

Likewise, a functional $F\left[\rho_{h}\right] \equiv \int_{D} \rho_{h} \phi d \mathbf{x}$ is needed, with $\phi \in \mathcal{Q}_{h}$ an arbitrary test function. Its functional derivative equals

$$
\frac{\delta F}{\delta \rho_{h}}=\phi
$$

Our starting point is to simply limit functionals in the Poisson bracket (प) on tessellation $\mathcal{I}_{h}$ to ones on the approximate finite element space, as follows

$$
\begin{aligned}
\frac{d F}{d t} & =[F, H] \\
& \equiv \sum_{K} \int_{K}\left(\frac{\delta H}{\delta \rho_{h}} \nabla_{h} \cdot \frac{\delta F}{\delta \mathbf{u}_{h}}-\frac{\delta F}{\delta \rho_{h}} \nabla_{h} \cdot \frac{\delta H}{\delta \mathbf{u}_{h}}-2 \Omega \times \frac{\delta H}{\delta \mathbf{u}_{h}} \cdot \frac{\delta F}{\delta \mathbf{u}_{h}}\right) d \mathbf{x}
\end{aligned}
$$

with element-wise differential operator $\nabla_{h}$. After integration by parts of the first two terms on the right-hand-side of (48) and introduction of numerical fluxes, we obtain

$$
\begin{gathered}
\frac{d F}{d t}=\sum_{K} \int_{K}\left(-\nabla_{h} \frac{\delta H}{\delta \rho_{h}} \cdot \frac{\delta F}{\delta \mathbf{u}_{h}}+\frac{\delta H}{\delta \mathbf{u}_{h}} \cdot \nabla_{h} \frac{\delta F}{\delta \rho_{h}}-2 \Omega \times \frac{\delta H}{\delta \mathbf{u}_{h}} \cdot \frac{\delta F}{\delta \mathbf{u}_{h}}\right) d K+ \\
\sum_{K} \int_{\partial K}\left(\frac{\delta H}{\delta \rho_{h}} \mathbf{n} \cdot \frac{\widehat{\delta F}}{\delta \mathbf{u}_{h}}-\frac{\widehat{\delta H}}{\delta \mathbf{u}_{h}} \cdot \mathbf{n} \frac{\delta F}{\delta \rho_{h}}\right) d \Gamma
\end{gathered}
$$

with element boundaries $\partial K$. Wide hats on the expressions in the boundary integrals indicate numerical fluxes. We chose the following numerical fluxes

$$
\frac{\widehat{\delta F}}{\delta \mathbf{u}_{h}}=(1-\theta) \frac{\delta F}{\delta \mathbf{u}_{h}^{L}}+\theta \frac{\delta F}{\delta \mathbf{u}_{h}^{R}} \quad \text { and } \quad \frac{\widehat{\delta H}}{\delta \mathbf{u}_{h}}=(1-\theta) \frac{\delta H}{\delta \mathbf{u}_{h}^{L}}+\theta \frac{\delta H}{\delta \mathbf{u}_{h}^{R}},
$$


where $L$ and $R$ indicate the traces from the left and right elements connected to the faces, and $0 \leq \theta \leq 1$. We emphasise the equivalence of the numerical fluxes used in (4.9) and in the FV discretisation for the case with constant basis and test functions on each element.

We use numerical fluxes (49b) and rewrite the sum over element boundaries into a sum over all faces. Together with (31), it yields the following DGFEM discretisation for linear, compressible Hamiltonian dynamics

$$
\begin{gathered}
\frac{d F}{d t}=\sum_{K} \int_{K}\left(-\nabla_{h} \frac{\delta H}{\delta \rho_{h}} \cdot \frac{\delta F}{\delta \mathbf{u}_{h}}+\frac{\delta H}{\delta \mathbf{u}_{h}} \cdot \nabla_{h} \frac{\delta F}{\delta \rho_{h}}-2 \Omega \times \frac{\delta H}{\delta \mathbf{u}_{h}} \cdot \frac{\delta F}{\delta \mathbf{u}_{h}}\right) d K+ \\
\sum_{e \in \Gamma_{i}} \int_{e}\left(\frac{\delta H}{\delta \rho_{h}^{L}}-\frac{\delta H}{\delta \rho_{h}^{R}}\right) \mathbf{n} \cdot\left((1-\theta) \frac{\delta F}{\delta \mathbf{u}_{h}^{L}}+\theta \frac{\delta F}{\delta \mathbf{u}_{h}^{R}}\right)+ \\
\left(\frac{\delta F}{\delta \rho_{h}^{R}}-\frac{\delta F}{\delta \rho_{h}^{L}}\right) \mathbf{n} \cdot\left(\frac{\delta H}{\delta \mathbf{u}_{h}^{R}} \theta+\frac{\delta H}{\delta \mathbf{u}_{h}^{L}}(1-\theta)\right) d \Gamma
\end{gathered}
$$

Here $\mathbf{n}=\mathbf{n}^{L}$ is the exterior normal vector connected with element $K^{L}$.

Technically speaking, periodic boundary conditions can be specified in ghost cells (denoted with subscript $R$ ), where values of the variables exactly coincide with the face-adjacent cell values (denoted with subscript $L_{p}$ ) at the other side of the periodic boundary

$$
\frac{\delta H}{\delta \mathbf{U}^{R}} \cdot \mathbf{n}=\frac{\delta H}{\delta \mathbf{U}^{L_{p}}} \cdot \mathbf{n} \quad \text { and } \quad \frac{\delta H}{\delta \rho^{R}}=\frac{\delta H}{\delta \rho^{L_{p}}}
$$

with $\mathbf{n}$ the normal to the boundary face. Geometrically speaking, there are of course only internal cells in a periodic domain. In the case of a threedimensional cuboid bounded by solid walls, the numerical fluxes on both the test functions and the Hamiltonian derivatives must vanish, cf. our specifications in (14). In terms of ghost cells, it implies that

$$
(1-\theta) \frac{\delta F}{\delta \mathbf{u}_{h}^{L}}+\theta \frac{\delta F}{\delta \mathbf{u}_{h}^{R}}=0 \quad \text { and } \quad(1-\theta) \frac{\delta H}{\delta \mathbf{u}_{h}^{L}}+\theta \frac{\delta H}{\delta \mathbf{u}_{h}^{R}}=0 \quad \text { at } \quad \Gamma_{D} .
$$

We will use, or rather assume, shortly that boundary conditions for incompressible flow should automatically satisfied by using Dirac's theory, given that those boundary conditions are satisfied for the discrete, compressible Hamiltonian discretisation.

By construction, the bracket (501) remains skew-symmetric. Unconventional is that the numerical flux is also acting on the test functions $\delta \mathcal{F} / \delta \mathbf{u}_{h}$. 
We refer to [45] for a proof that the bracket (50) can be transformed to a classical, discontinuous Galerkin finite element weak formulation with alternating fluxes, provided $\theta=1 / 2$ at boundary faces and for constant material parameters. When material parameters are a function of space, then the Hamiltonian formulation with its division between bracket and Hamiltonian becomes crucial. Not only the skew-symmetric or alternating fluxes matter then but also the dual, Hamiltonian projection. The DG discretisation with a polynomial approximation of order zero will exactly coincide with our FV discretisation. Note that for $\theta=1 / 2$ the well-known image boundary condition emerges from (52). We emphasise, though, that for $\theta \neq 1 / 2$ our general condition (52) still applies, but that it seems no longer quite equivalent to the standard, alternating flux formulation applied directly to the PDEs.

Variables are expanded on each element $K$ in terms of local basis functions such that: $\mathbf{u}_{h}=\phi_{\beta} \mathbf{u}_{\beta}$ and $\rho_{h}=\phi_{\beta} \rho_{\beta}$. Both coefficients and test functions require elemental superscripts, which we silently omit. Greek numerals are used locally on each element $K$ and we apply the summation convention over repeated indices. Variational and function derivatives are then related as follows

$$
\begin{aligned}
\delta \mathcal{F} & =\sum_{K} \int_{K} \frac{\delta \mathcal{F}}{\delta \mathbf{u}_{h}} \delta \mathbf{u}_{h}+\frac{\delta \mathcal{F}}{\delta \rho_{h}} \delta \rho_{h} d K \\
& =\sum_{K}\left(\int_{K} \frac{\delta \mathcal{F}}{\delta \mathbf{u}_{h}} \phi_{\beta} d K\right) \delta \mathbf{u}_{\beta}+\left(\int_{K} \frac{\delta \mathcal{F}}{\delta \rho_{h}} \phi_{\beta} d K\right) \delta \rho_{\beta} \\
& =\sum_{K} \frac{\partial F}{\partial \mathbf{u}_{\beta}} \delta \mathbf{u}_{\beta}+\frac{\partial F}{\partial \rho_{\beta}} \delta \rho_{\beta} .
\end{aligned}
$$

Similarly, by starting from (530) and using the relation

$$
M_{\alpha \beta} \mathbf{u}_{\beta}=\int_{K} \phi_{\alpha} \mathbf{u}_{h} d K
$$

with local mass matrix $M_{\alpha \beta}=M_{\alpha \beta}^{K}$, one can derive

$$
\frac{\delta \mathcal{F}}{\delta \mathbf{u}_{h}}=M_{\beta \gamma}^{-1} \frac{\partial F}{\partial \mathbf{u}_{\beta}} \phi_{\gamma} \quad \text { and } \quad \frac{\delta \mathcal{F}}{\delta \rho_{h}}=M_{\beta \gamma}^{-1} \frac{\partial F}{\partial \rho_{\beta}} \phi_{\gamma} .
$$

By substitution of (55) into (50), we immediately derive the desired form of 
the finite-dimensional Hamiltonian discretisation

$$
\begin{gathered}
\frac{d F}{d t}=\sum_{K}\left(\frac{\partial H}{\partial \mathbf{u}_{\beta}} \frac{\partial F}{\partial \rho_{\alpha}}-\frac{\partial F}{\partial \mathbf{u}_{\beta}} \frac{\partial H}{\partial \rho_{\alpha}}\right) \cdot \mathbf{E}_{\gamma \mu} M_{\beta \gamma}^{-1} M_{\alpha \mu}^{-1}-2 \Omega \times \frac{\partial H}{\partial \mathbf{u}_{\alpha}} \cdot \frac{\partial F}{\partial \mathbf{u}_{\beta}} M_{\alpha \beta}^{-1} \\
+\sum_{e \in \Gamma_{i}}(1-\theta)\left(\frac{\partial H}{\partial \rho_{\alpha}^{L}} \frac{\partial F}{\partial \mathbf{u}_{\beta}^{L}}-\frac{\partial F}{\partial \rho_{\alpha}^{L}} \frac{\partial H}{\partial \mathbf{u}_{\beta}^{L}}\right) \cdot \mathbf{G}_{\gamma \mu}^{L L} M_{\alpha \mu}^{-L} M_{\beta \gamma}^{-L} \\
+\theta\left(\frac{\partial H}{\partial \rho_{\alpha}^{L}} \frac{\partial F}{\partial \mathbf{u}_{\beta}^{R}}-\frac{\partial F}{\partial \rho_{\alpha}^{L}} \frac{\partial H}{\partial \mathbf{u}_{\beta}^{R}}\right) \cdot \mathbf{G}_{\gamma \mu}^{L R} M_{\alpha \mu}^{-L} M_{\beta \gamma}^{-R} \\
-(1-\theta)\left(\frac{\partial H}{\partial \rho_{\alpha}^{R}} \frac{\partial F}{\partial \mathbf{u}_{\beta}^{L}}-\frac{\partial F}{\partial \rho_{\alpha}^{R}} \frac{\partial H}{\partial \mathbf{u}_{\beta}^{L}}\right) \cdot \mathbf{G}_{\gamma \mu}^{R L} M_{\alpha \mu}^{-R} M_{\beta \gamma}^{-L} \\
-\theta\left(\frac{\partial H}{\partial \rho_{\alpha}^{R}} \frac{\partial F}{\partial \mathbf{u}_{\beta}^{R}}-\frac{\partial F}{\partial \rho_{\alpha}^{R}} \frac{\partial H}{\partial \mathbf{u}_{\beta}^{R}}\right) \cdot \mathbf{G}_{\gamma \mu}^{R R} M_{\alpha \mu}^{-R} M_{\beta \gamma}^{-R}
\end{gathered}
$$

with elemental (vector) matrices $\mathbf{E}_{\gamma \mu}$ and $\mathbf{G}_{\gamma \mu}^{L R}$ etc. These read

$$
\mathbf{E}_{\gamma \mu}=\int_{K} \phi_{\gamma} \nabla_{h} \phi_{\mu} d K \quad \text { and } \quad \mathbf{G}_{\gamma \mu}^{L R}=\int_{e} \mathbf{n} \phi_{\mu}^{L} \phi_{\gamma}^{R} d \Gamma
$$

with similar relations for other terms. Finally, after substitution of (5.5) into Hamiltonian (44), it becomes

$$
H=\frac{1}{2} \sum_{K} M_{\alpha \beta}\left(\mathbf{u}_{\alpha} \cdot \mathbf{u}_{\beta}+\rho_{\alpha} \rho_{\beta}\right)
$$

A global formulation is useful for the incompressible case. We therefore introduce a reordering into global coefficients $\mathbf{U}_{i}=\mathbf{U}_{i}(t)=(U, V, W)_{i}^{T}$ and $R_{k}(t)$, instead of the elemental ones, in the finite element expansion of $\mathbf{u}_{h}$ and $\rho_{h}$ with indices running over their respective, global ranges. It turns out that the local matrices $M_{\alpha \beta}$ and $\mathbf{E}_{\gamma \mu}$ in (56) and (58) readily extend to global matrices $M_{i j}$ and $\mathbf{E}_{i j}$. These have a block structure in which each elemental matrix fits in separation from the others. The contribution of the numerical fluxes lead to coupling between the elements, which can be incorporated into a global matrix $\mathbf{G}_{i j}$. The latter is straightforwardly defined computationally by a loop over the faces, and we will therefore not provide an explicit expression. The resulting, global Hamiltonian formulation then 
becomes

$$
\begin{aligned}
\frac{d F}{d t}=[F, H]_{d} \equiv & \left(\frac{\partial H}{\partial \mathbf{U}_{j}} \frac{\partial F}{\partial R_{i}}-\frac{\partial F}{\partial \mathbf{U}_{j}} \frac{\partial H}{\partial R_{i}}\right) \cdot \mathbf{D I V}_{k l} M_{i k}^{-1} M_{j l}^{-1} \\
& -2 \Omega \times \frac{\partial H}{\partial \mathbf{U}_{i}} \cdot \frac{\partial F}{\partial \mathbf{U}_{j}} M_{i j}^{-1}
\end{aligned}
$$

with the divergence vector operator $\mathbf{D I V} \mathbf{V}_{k l} \equiv \mathbf{E}_{k l}-\mathbf{G}_{k l}$ and global Hamiltonian

$$
H=\frac{1}{2} M_{i j}\left(\mathbf{U}_{i} \cdot \mathbf{U}_{j}+R_{i} R_{j}\right)
$$

The resulting equations of motion arising from (59) are

$$
\begin{aligned}
\dot{\mathbf{U}}_{j} & =-M_{j k}^{-1} R_{l} \mathbf{D I V}_{k l}-2 \boldsymbol{\Omega} \times \mathbf{U}_{j} \\
M_{k l} \dot{R}_{l} & =\mathbf{U}_{j} \cdot \mathbf{D I V}_{j k}
\end{aligned}
$$

with the dot denoting a time derivative.

\subsubsection{Hamiltonian DGFEM discretisation for linearised incompressible flow}

In close analogy with the continuous case and the FV-case, Dirac's theory is applied to the Hamiltonian dynamics (5.9). The density expansion coefficients are all restricted in every local element such that the resulting density in the element is zero. The following primary constraints are imposed on the discrete density field

$$
D_{k}=M_{k l} R_{l}
$$

Preservation of the constraints in time leads to the following consistency relation

$$
0=\dot{D}_{k}=\left[D_{k}, H\right]_{d}+\lambda_{l}\left[D_{k}, D_{l}\right]_{d}
$$

Using (5]) in the bracket (5.9a) shows that $\left[D_{k}, D_{l}\right]_{d}=0$. The Lagrange multipliers $\lambda_{l}$ in (62) thus remain undetermined, but the consistency requirement gives rise to secondary constraints $L_{k}=\left[D_{k}, H\right]_{d}=0$. Analogous to the continuous and FV-case, the secondary constraint is the discrete version of the divergence-free velocity field property (ㅁ). To wit

$$
L_{k}=\left[D_{k}, H\right] \equiv \mathbf{D I V}_{l k} \cdot \mathbf{U}_{l}
$$


with the discrete divergence operator $\mathbf{D I V}_{l k}$. We start again with both primary constraints and require these to remain preserved under the Hamiltonian dynamics. We obtain

$$
\begin{aligned}
0 & =\dot{D}_{k}=\left[D_{k}, H\right]_{d}+\mu_{l}\left[D_{k}, L_{l}\right]_{d}, \\
0 & =\dot{L}_{k}=\left[L_{k}, H\right]_{d}+\lambda_{l}\left[L_{k}, D_{l}\right]_{d}+\mu_{l}\left[L_{k}, L_{l}\right]_{d} .
\end{aligned}
$$

Application of the primary constraint implies that $L_{k}=\left[D_{k}, H\right]=0$ in (64a) . Hence,

$$
\mu_{l}\left[D_{k}, L_{l}\right]=\mu_{l} \mathbf{D I V} \mathbf{V}_{m l} M_{j m}^{-1} \cdot \mathbf{D I V} \mathbf{V}_{j k}=0 .
$$

The matrix acting on $\mu_{l}$ is a discrete Laplacian. It is nonsingular, whence $\mu_{l}=0$. Consequently, (64b) reduces to

$$
\begin{aligned}
0=\dot{L}_{k} & =\left[L_{k}, H\right]_{d}+\lambda_{l}\left[L_{k}, D_{l}\right]_{d} \\
& =-\mathbf{D I V}_{j k} \cdot 2 \Omega \times \mathbf{U}_{j}-\lambda_{l} \mathbf{D I V}_{j k} M_{j m}^{-1} \cdot \mathbf{D I V}_{m l},
\end{aligned}
$$

which is the discrete equivalent of the Poisson equation in (29) . Finally, the resulting discrete, linear, incompressible Hamiltonian dynamics is given by

$$
\frac{d F}{d t}=[F, H]_{i n c} \equiv-\frac{\partial F}{\partial \mathbf{U}_{j}} \cdot\left(2 \Omega \times \frac{\partial H}{\partial \mathbf{U}_{i}} M_{i j}^{-1}+\lambda_{l} M_{j k}^{-1} \mathbf{D I} \mathbf{V}_{k l}\right)
$$

with energy function

$$
H=\frac{1}{2} M_{i j} \mathbf{U}_{i} \cdot \mathbf{U}_{j} .
$$

The final system consists of the ordinary differential equations following directly from (67) after using $F=\mathbf{U}_{j}$, as follows

$$
\dot{\mathbf{U}}_{j}=-2 \Omega \times \mathbf{U}_{j}-\lambda_{l} M_{j k}^{-1} \mathbf{D I V} \mathbf{V}_{k l}
$$

combined with (66):

$$
\lambda_{l} \mathbf{D I V}_{j k} M_{j m}^{-1} \cdot \mathbf{D I V} \mathbf{V}_{m l}=-\mathbf{D I V}_{j k} \cdot 2 \Omega \times \mathbf{U}_{j}
$$

\section{Time Integrator}

We consider a symplectic time integrator for the time discretisation of linear compressible (60) and incompressible (68) Hamiltonian dynamics. Symplectic time integrators form the subclass of geometric integrators which, by definition, are canonical transformations. The modified midpoint time integrator was chosen amongst other symplectic schemes [18]. It is implicit, which requires more computation, but that pays off in dealing with the momentum and continuity equations in a rotating frame of reference. 


\subsection{Linear, compressible flow}

Applying the modified midpoint scheme to the discrete compressible Hamiltonian dynamics (5.9) or (50), one gets

$$
\begin{aligned}
& \frac{\mathbf{U}_{j}^{n+1}-\mathbf{U}_{j}^{n}}{\Delta t}=-M_{j k}^{-1} \frac{\left(R_{l}^{n+1}+R_{l}^{n}\right)}{2} \mathbf{D I V}_{k l}-\boldsymbol{\Omega} \times\left(\mathbf{U}_{j}^{n+1}+\mathbf{U}_{j}^{n}\right) \\
& M_{k l} \frac{\left(R_{l}^{n+1}-R_{l}^{n}\right)}{\Delta t}=\frac{\mathbf{U}_{j}^{n+1}+\mathbf{U}_{j}^{n}}{2} \cdot \mathbf{D I V}_{j k} .
\end{aligned}
$$

Proposition 1. The numerical scheme for linear, compressible fluid flow given by $(\mathbf{⿴ 囗 ⿰ 丿 ㇄ 丁 ) ~})$ is exactly energy conserving, such that $M_{i j}\left(\mathbf{U}_{i}^{n+1} \cdot \mathbf{U}_{j}^{n+1}+\right.$ $\left.R_{i}^{n+1} R_{j}^{n+1}\right)=M_{i j}\left(\mathbf{U}_{i}^{n} \cdot \mathbf{U}_{j}^{n}+R_{i}^{n} R_{j}^{n}\right)$.

Proof. Multiply equations (四) with $M_{i j} \mathbf{U}_{i}^{n+1}, R_{k}^{n+1}$ and $M_{i j} \mathbf{U}_{i}^{n}, R_{k}^{n}$. Thereafter, add them up. After some manipulation, the Hamiltonian on the $(n+1)$ th time level can be shown to equal the Hamiltonian on the $n$-th level.

\subsection{Incompressible flow}

The midpoint time integrator is also applied to the incompressible discrete Hamiltonian dynamics (67) or ([6]), giving

$$
\begin{aligned}
& \frac{\left(\mathbf{U}_{j}^{n+1}-\mathbf{U}_{j}^{n}\right)}{\Delta t}=-\boldsymbol{\Omega} \times\left(\mathbf{U}_{j}^{n+1}+\mathbf{U}_{j}^{n}\right)-\lambda_{l}^{n+1} M_{j k}^{-1} \mathbf{D I V}_{k l} \\
& \lambda_{l}^{n+1} \mathbf{D I V}_{j k} M_{j m}^{-1} \cdot \mathbf{D I V} \mathbf{V}_{m l}=-\mathbf{D I V}_{j k} \cdot \boldsymbol{\Omega} \times\left(\mathbf{U}_{j}^{n+1}+\mathbf{U}_{j}^{n}\right) .
\end{aligned}
$$

Proposition 2. The numerical scheme for linear, incompressible fluid flow given by $(\mathbb{}[)$ exactly conserves energy and the discrete zero-divergence property in time, such that $\mathbf{D I V} \mathbf{V}_{j m} \cdot \mathbf{U}_{j}^{n+1}=\mathbf{0}$ given that $\mathbf{D I V} \mathbf{V}_{j m} \cdot \mathbf{U}_{j}^{0}=\mathbf{0}$ and $M_{i j} \mathbf{U}_{i}^{n+1} \cdot \mathbf{U}_{j}^{n+1}=M_{i j} \mathbf{U}_{i}^{n} \cdot \mathbf{U}_{j}^{n}$.

Proof. Firstly, we present the proof for the conservation of the discrete zerodivergence, under the assumption that the current velocity of the $n^{\text {th }}$-time level is discretely divergence free, i.e., $L_{m}=\mathbf{D I V}_{j m} \cdot \mathbf{U}_{j}^{n}=0$. We apply the discrete divergence operator on both sides of ( $\square \mathrm{a})$ and use that the present velocity is divergence free, to obtain

$$
\begin{aligned}
\mathbf{D I V}_{j m} \cdot \mathbf{U}_{j}^{n+1} / \Delta t= & -\mathbf{D I V}_{j m} \cdot \boldsymbol{\Omega} \times\left(\mathbf{U}_{j}^{n+1}+\mathbf{U}_{j}^{n}\right) \\
& -\mathbf{D I V}_{j m} \cdot \lambda_{l}^{n+1} M_{j k}^{-1} \mathbf{D I V}_{k l}
\end{aligned}
$$


The right hand side of ([י2) exactly coincides with ([16) and therefore $\mathbf{D I V}_{j m}$. $\mathbf{U}_{j}^{n+1}=\mathbf{0}$.

Secondly, energy conservation means that the discrete Hamiltonian energy functional (G7b) stays unchanged in time. Multiplication of (ㅁa) with $M_{i j} \mathbf{U}_{i}^{n+1}$ and $M_{i j} \mathbf{U}_{i}^{n}$, followed by summation of both equations yields

$$
M_{i j} \frac{\left(\mathbf{U}_{i}^{n+1} \cdot \mathbf{U}_{j}^{n+1}-\mathbf{U}_{i}^{n} \cdot \mathbf{U}_{j}^{n}\right)}{\Delta t}=-\lambda_{l}^{n+1} \mathbf{D I V}_{i l} \cdot\left(\mathbf{U}_{i}^{n+1}+\mathbf{U}_{i}^{n}\right)=0,
$$

since the terms involving rotational effects cancel and in the last step we use that present and future time velocities are divergence free, as shown in the first part of this proof. Hence, the difference of the energy at the $(n+1)^{\text {th }}$ and $n^{\text {th }}$ level is zero.

\subsection{Initial conditions}

As proven above, the discontinuous Galerkin discretisation for linearised incompressible fluid flow conserves energy and is divergence free at the discrete level. The proofs require a discrete, divergence-free initial condition, i.e., $\mathbf{D I V}_{j m} \cdot \mathbf{U}_{j}^{0}=0$. This condition is not guaranteed automatically, since the projection of the initial, divergence-free velocity field on the chosen discontinuous Galerkin finite element space only satisfies discrete zerodivergence up to the order of accuracy. We therefore require a preprocessing step on this projected velocity $\mathbf{U}$. We are looking for a $\mathbf{U}^{*}$ for which $\mathbf{D I V}_{j m} \cdot \mathbf{U}_{j}^{*}=0$ exactly and $\left\|\mathbf{U}^{*}-\mathbf{U}\right\|$ is minimal. Note that the matrix DIV $_{j m}$ is not square. Hereafter, we denote the associated, global matrix by $D I V$ and the vector of velocity unknowns as $\mathbf{U}$ (so without indices). Basically, the latter problem transforms into a well-known problem in vector calculus: find a projection of the vector $\mathbf{U}$ on the space $A$ : the null-space of discrete divergence matrix operator $D I V$, defined as

$$
A=\left\{\mathbf{Q} \in \mathbb{R}^{3 N_{\text {dof }}}: \quad D I V \mathbf{Q}=0\right\}
$$

with $N_{\text {dof }}$ the degrees of freedom per velocity component (assuming them to be equal for simplicity).

From linear algebra [14, [13], we obtain that the closest vector from the $A$-space will be the projection of vector $\mathbf{U}$ on the space, which is

$$
\mathbf{U}^{*} \equiv \operatorname{proj}_{A} \mathbf{U}=\mathbf{U}+\mathbf{U}_{\perp} .
$$




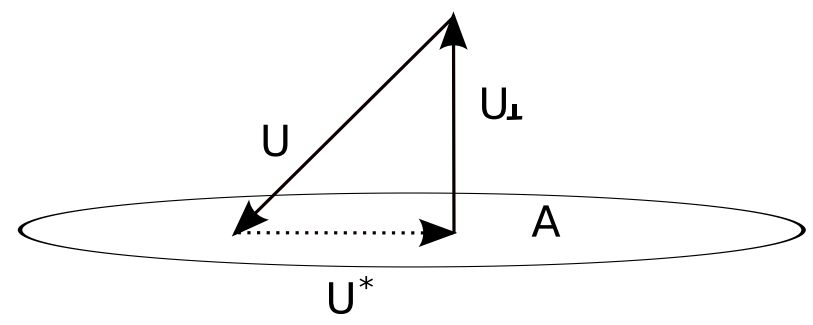

Figure 1: Projection of vector $\mathbf{U}$ on the null-space of matrix $D I V$.

Applying the $D I V$-operator on ([75), we find

$$
0=D I V \mathbf{U}^{*}=D I V \mathbf{U}+D I V \mathbf{U}_{\perp}
$$

which results in

$$
D I V \mathbf{U}_{\perp}=-D I V \mathbf{U}
$$

The latter equation is solved for $\mathbf{U}_{\perp}$ via a least-square approximation [I6] up to machine precision. Hence, the projected velocity is preprocessed using (75), and the resulting velocity field has become exactly discrete divergence free, as required.

\subsection{Other properties of the algebraic system}

A direct DGFEM discretisation of the incompressible Navier-Stokes equations (or the Euler equations as special case) generally requires the inf-sup condition to be satisfied to attain numerical stability [15, 27]. In order to get a stable pressure approximation, two different strategies are often pursued: either a pressure stabilisation term is used or the approximation spaces for velocity and pressure are chosen (differently) such that an inf-sup compatibility condition is fulfilled. Nonetheless, our numerical discretisation for linear, incompressible flow does not suffer from those drawbacks. The exact preservation of the Hamiltonian dynamics as well as the constraints makes the system unconditionally stable.

Furthermore, the three-dimensionality of the problem results in a large algebraic system, which we represent using the sparse matrix structures available in PETSc [38, 39]. Figure [ 2 shows the sparsity of a matrix, needed to determine $\mathbf{U}_{j}^{n+1}$ and $\lambda_{l}^{n+1}$ in the discretisation for incompressible flow ([0). We use a linear, iterative solver to converge to the desired tolerance. To improve the convergence rate of the iterative solver ILU preconditioners were used with controlled memory usage of the resulting algebraic system. 


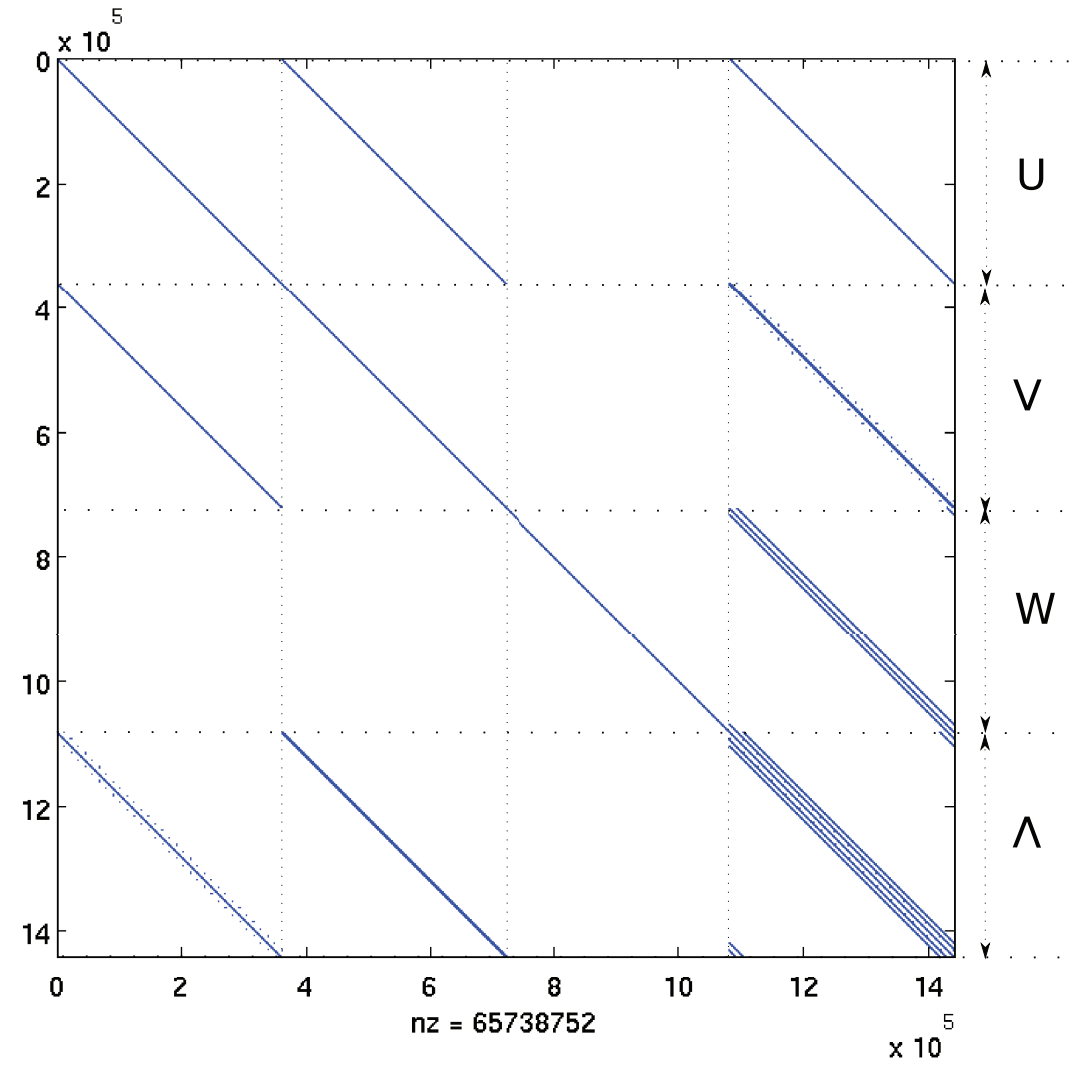

Figure 2: A structure of the resulting square sparse matrix with more than $10^{8}$ non-zero elements and $\Omega_{1}=\Omega_{2}=0$, and $\Omega_{3}=1$. $\Lambda$ denotes the vector of unknown Lagrange multipliers. 


\section{Tests of numerical scheme}

Discretisations for linear compressible and incompressible flows were implemented for discontinuous Galerkin methods in the hpGEM C++ software framework [32]. The developed applications are consequently highly objectoriented, easy to maintain and extend. Although the tests considered concern cuboids, the implementation can cope with general geometries and meshes. The three-dimensionality of the problem poses, however, significant requirements on speed and memory use. The sparse matrix data structures available in the PETSc library are therefore used. An ILU pre-conditioner is applied to the linear algebraic system before applying a GMRES linear solver [38, 3.9]. The number of iterations varies for the different test cases and strongly depends on the dimensions of the algebraic system, e.g., the grid size and the amount of basis functions. In the case of quadratic polynomial basis functions with a grid of $64 \times 64 \times 64$ elements, which is the computationally most demanding case, one needs approximately forty GMRES iterations to reach the tolerance $10^{-14}$ in solving the algebraic equation for incompressible flow.

Although the main goal is to simulate inertial waves in a rectangular box, several extra test cases were performed to verify the approaches and techniques used. Two text cases (periodic waves and waves in a domain with no-slip boundaries) for the compressible fluid were implemented, tested and validated by comparison with an available exact solutions. Additionally, an attempt has been made to attain energy attractors in the domain with a geometrical asymmetry. In all tests presented, $\theta=1 / 2$ was used in the numerical flux. Other values of $0 \leq \theta \leq 1$ were also used for various test cases with similar results.

\subsection{Compressible harmonic waves in a periodic domain}

Consider linear, compressible fluid flow with zero rotation $\Omega=(0,0,0)$ in a rectangular triple periodic domain $D=[0,1]^{3}$. The following expressions satisfy the linear Euler equations

$$
\begin{aligned}
u & =A_{1} \cos \left(2 k_{x}(t-x)\right), \\
v & =A_{2} \cos \left(2 k_{y}(t-y)\right), \\
w & =A_{3} \cos \left(2 k_{z}(t-z)\right), \\
\rho & \left.=A_{1} \cos \left(2 k_{x}(t-x)\right)+A_{2} \cos \left(2 k_{y}(t-y)\right)+A_{3} \cos 2 k_{z}(t-z)\right) .
\end{aligned}
$$

Each component of the velocity vector is a traveling wave in the direction of the corresponding axes. The numerical discretisation is initialised with 
(78) at $t=0, k_{x}=k_{y}=k_{z}=2 \pi$ and $A_{1}=A_{2}=A_{3}=1$. Numerical and exact solutions were compared during several periods. Figure 3 presents the numerical density profile during one time period of the traveling waves. Discrete energy is conserved up to machine precision even after one hundred

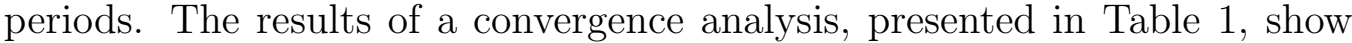
that the numerical scheme is first, second and third order accurate in space for, respectively constant, linear and quadratic polynomial approximations.

Table 1: Convergence of the error for compressible traveling waves in a thrqee-dimensional periodic domain. Due to symmetry all velocity components have the same error.

\begin{tabular}{ll|ll|ll|ll}
\hline \hline & & \multicolumn{2}{|c|}{$p=0$} & \multicolumn{2}{c|}{$p=1$} & \multicolumn{2}{c}{$p=2$} \\
\hline Grid & & $l^{2}$-error & order & $l^{2}$-error & order & $l^{2}$-error & order \\
\hline $4 \times 4 \times 4$ & $\mathbf{u}$ & $1.011 \mathrm{e}+0$ & - & $4.3024 \mathrm{E}-1$ & - & $1.8532 \mathrm{E}-2$ & - \\
& $\rho$ & $1.751 \mathrm{e}+0$ & - & $7.4520 \mathrm{E}-1$ & - & $3.2098 \mathrm{E}-2$ & - \\
\hline $8 \times 8 \times 8$ & $\mathbf{u}$ & $8.249 \mathrm{E}-1$ & 0.3 & $1.3087 \mathrm{E}-1$ & 1.7 & $3.0944 \mathrm{E}-3$ & 2.6 \\
& $\rho$ & $1.428 \mathrm{e}+0$ & 0.3 & $2.2667 \mathrm{E}-1$ & 1.7 & $5.3598 \mathrm{E}-3$ & 2.6 \\
\hline \multirow{2}{*}{$16 \times 16 \times 16$} & $\mathbf{u}$ & $2.405 \mathrm{E}-1$ & 1.8 & $2.9192 \mathrm{E}-2$ & 2.1 & $3.4723 \mathrm{E}-4$ & 3.2 \\
& $\rho$ & $4.166 \mathrm{E}-1$ & 1.8 & $5.0554 \mathrm{E}-2$ & 2.1 & $6.0142 \mathrm{E}-4$ & 3.2 \\
\hline $32 \times 32 \times 32$ & $\mathbf{u}$ & $7.193 \mathrm{E}-2$ & 1.7 & $5.0432 \mathrm{E}-3$ & 2.5 & $2.9366 \mathrm{E}-5$ & 3.6 \\
& $\rho$ & $1.245 \mathrm{E}-1$ & 1.7 & $8.7321 \mathrm{E}-3$ & 2.5 & $5.0864 \mathrm{E}-5$ & 3.6 \\
\hline $64 \times 64 \times 64$ & $\mathbf{u}$ & $2.638 \mathrm{E}-2$ & 1.5 & $1.0813 \mathrm{E}-3$ & 2.2 & $1.8266 \mathrm{E}-6$ & 4.0 \\
& $\rho$ & $4.579 \mathrm{E}-2$ & 1.4 & $2.3901 \mathrm{E}-3$ & 1.8 & $3.1638 \mathrm{E}-6$ & 4.0 \\
\hline
\end{tabular}

\subsection{Compressible waves with slip-flow boundary conditions}

Next, linear, acoustic fluid flow is considered in domain $D$, but now slipflow boundary conditions are used with zero normal component of the velocity field at domain boundaries. Boundary conditions are effectively implemented with the help of ghost cells, where the values of velocity and density fields are specified to satisfy the boundary conditions. One can check that

$$
\begin{aligned}
u & =-A_{1} \sin \left(2 k_{x} x\right) \cos (\omega t), \\
v & =-A_{2} \sin \left(2 k_{y} y\right) \cos (\omega t) \\
w & =-A_{3} \sin \left(2 k_{z} z\right) \cos (\omega t) \\
\rho & =A_{1} \sin \left(2 k_{x} x\right) \cos (\omega t)+A_{2} \sin \left(2 k_{y} y\right) \cos (\omega t)+A_{3} \sin \left(2 k_{z} z\right) \cos (\omega t) .
\end{aligned}
$$

exactly satisfy the linearised compressible Euler equations with slip-flow boundary conditions. The numerical scheme is initialised using ([तQ) at $t=0$, where $\omega=k_{x}=k_{y}=k_{z}=2 \pi$ and $A_{1}=A_{2}=A_{3}=1$. Figure $⿴$ shows the 


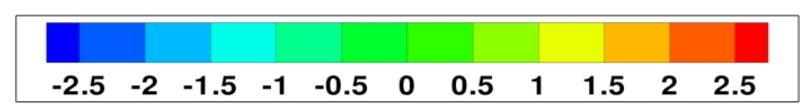

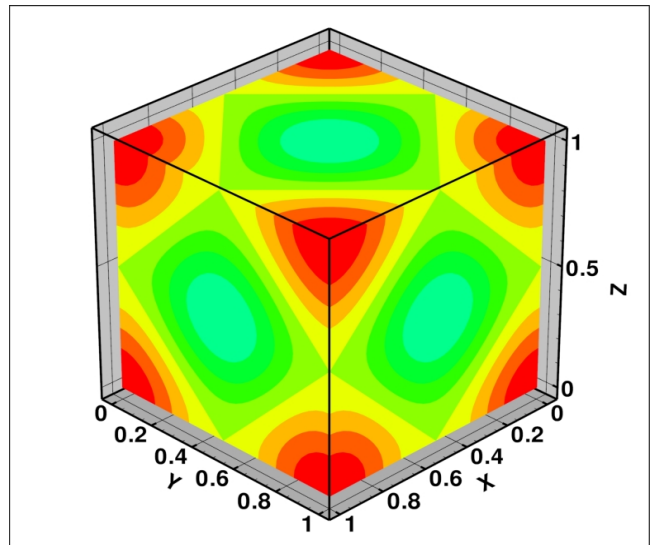

(a) Density profile at $\mathrm{t}=0$

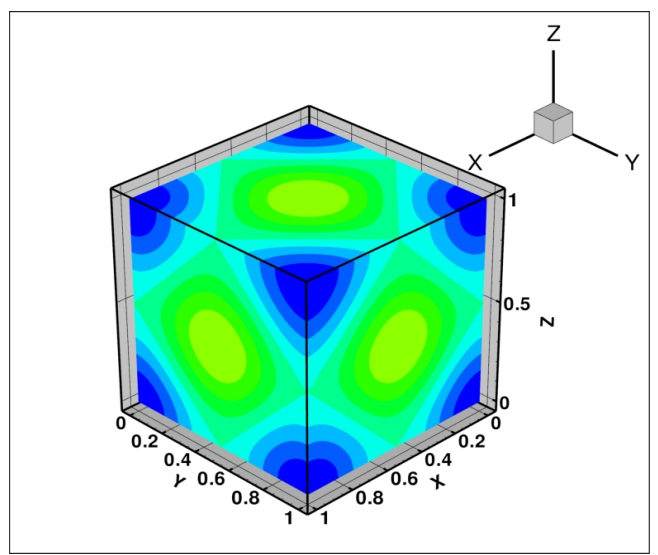

(c) Density profile at $\mathrm{t}=\mathrm{T} / 2$

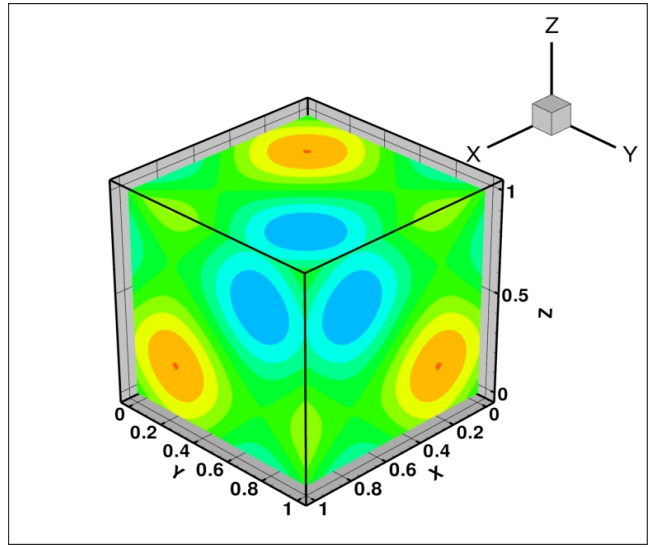

(b) Density profile at $\mathrm{t}=\mathrm{T} / 4$

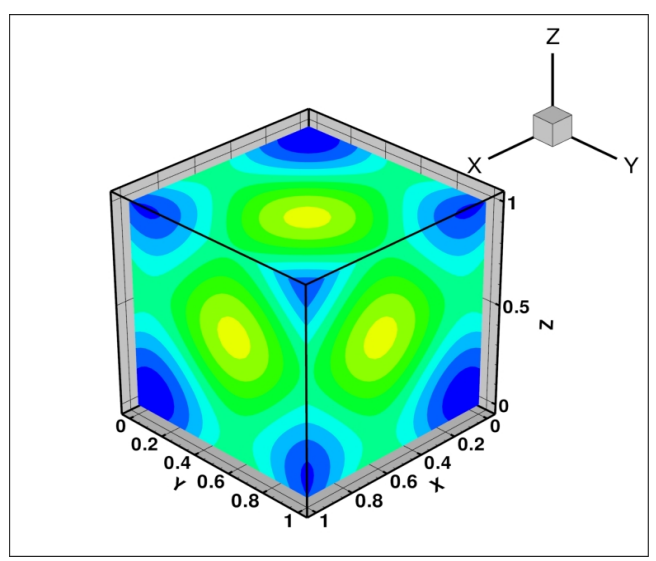

(d) Density profile at $t=3 \mathrm{~T} / 4$

Figure 3: Plots of the density field computed with the discretised compressible Hamiltonian formulation. A $32 \times 32 \times 32$ grid with time step $\Delta t=T / 20$, where $T$ is the time period of the harmonic waves, was used in a periodic domain. 
numerical density profile during a full time period. Discrete energy again is conserved up to machine precision, after one hundred wave periods. A convergence analysis is given in Table 2 .

Table 2: Convergence of the error for compressible standing waves in a cuboid with solid walls. Due to symmetry all velocity components have the same error.

\begin{tabular}{ll|ll|ll|ll}
\hline \hline & & \multicolumn{2}{|c|}{$p=0$} & & \multicolumn{2}{c|}{$p=1$} & \multicolumn{2}{c}{$p=2$} \\
\hline Grid & & $l^{2}$-error & order & $l^{2}$-error & order & $l^{2}$-error & order \\
\hline $4 \times 4 \times 4$ & $\mathbf{u}$ & $7.909 \mathrm{E}-1$ & - & $1.6871 \mathrm{E}-1$ & - & $7.6351 \mathrm{E}-3$ & - \\
& $\rho$ & $2.391 \mathrm{e}+0$ & - & $6.8551 \mathrm{E}-1$ & - & $2.9242 \mathrm{E}-2$ & - \\
\hline $8 \times 8 \times 8$ & $\mathbf{u}$ & $5.012 \mathrm{E}-1$ & 0.7 & $6.8455 \mathrm{E}-2$ & 1.3 & $3.2262 \mathrm{E}-4$ & 4.5 \\
& $\rho$ & $1.134 \mathrm{e}+0$ & 0.6 & $1.9319 \mathrm{E}-1$ & 1.8 & $5.3301 \mathrm{E}-3$ & 2.5 \\
\hline \multirow{2}{*}{$16 \times 16 \times 16$} & $\mathbf{u}$ & $8.794 \mathrm{E}-2$ & 2.5 & $6.2392 \mathrm{E}-3$ & 3.4 & $4.2721 \mathrm{E}-5$ & 3.0 \\
& $\rho$ & $3.877 \mathrm{E}-1$ & 1.4 & $4.9391 \mathrm{E}-2$ & 2.0 & $5.9395 \mathrm{E}-4$ & 3.2 \\
\hline $32 \times 32 \times 32$ & $\mathbf{u}$ & $4.013 \mathrm{E}-2$ & 1.1 & $1.0702 \mathrm{E}-3$ & 2.5 & $5.6751 \mathrm{E}-6$ & 4.6 \\
& $\rho$ & $1.033 \mathrm{E}-1$ & 1.9 & $8.5331 \mathrm{E}-3$ & 2.5 & $5.0721 \mathrm{E}-5$ & 3.5 \\
\hline \multirow{2}{*}{$64 \times 64 \times 64$} & $\mathbf{u}$ & $2.003 \mathrm{E}-2$ & 1.0 & $2.5982 \mathrm{E}-4$ & 2.0 & $7.0557 \mathrm{E}-7$ & 3.0 \\
& $\rho$ & $2.973 \mathrm{E}-2$ & 1.8 & $2.3531 \mathrm{E}-3$ & 1.9 & $5.1638 \mathrm{E}-6$ & 3.1 \\
\hline
\end{tabular}

\subsection{Incompressible waves in a periodic domain}

The compressible test cases were mainly interesting as a quality assurance step for linearised incompressible fluid flow, which we consider next. An exact solution was found for the linear, incompressible, rotational Euler equations (22.9) with periodic boundary conditions

$$
\begin{aligned}
& u=\frac{1}{2 \pi}\left[\sqrt{3} \cos \left(2 \pi(x+y+z)+\frac{\sqrt{3}}{3} t\right)+3 \sin \left(2 \pi(x+y+z)+\frac{\sqrt{3}}{3} t\right)\right], \\
& v=\frac{1}{2 \pi}\left[\sqrt{3} \cos \left(2 \pi(x+y+z)+\frac{\sqrt{3}}{3} t\right)-3 \sin \left(2 \pi(x+y+z)+\frac{\sqrt{3}}{3} t\right)\right], \\
& w=-\frac{1}{\pi} \sqrt{3} \cos \left(2 \pi(x+y+z)+\frac{\sqrt{3}}{3} t\right), \\
& P=\frac{1}{2 \pi^{2}} \cos \left(2 \pi(x+y+z)+\frac{\sqrt{3}}{3} t\right),
\end{aligned}
$$

where the rotation vector is $\boldsymbol{\Omega}=(0,0,1)$ and $P$ is the pressure. This exact solution is used for the initialisation in a periodic domain $D=[0,1]^{3}$. As was already discussed, the Lagrange multiplier $\lambda=P$ plays the role of the pressure in our incompressible Hamiltonian discretisation. The numerical velocity and pressure fields are compared against the exact solution during 


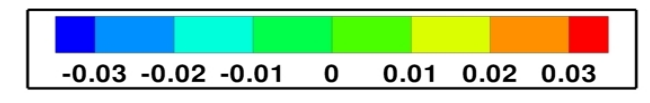

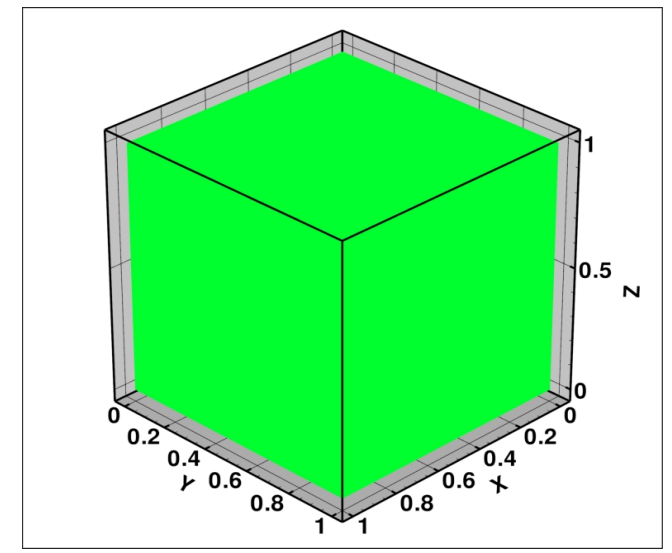

(a) Density profile at $\mathrm{t}=0$

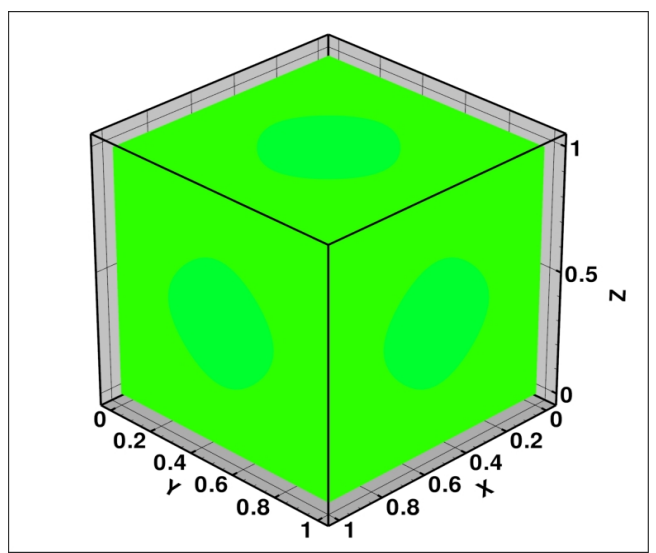

(c) Density profile at $\mathrm{t}=\mathrm{T} / 2$

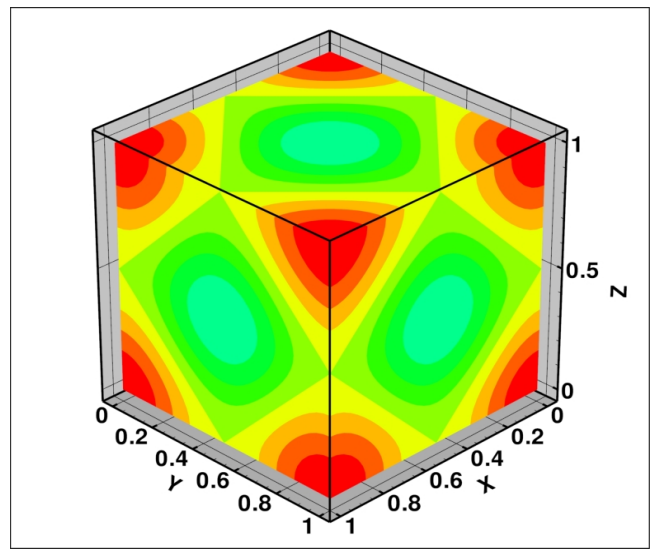

(b) Density profile at $\mathrm{t}=\mathrm{T} / 4$

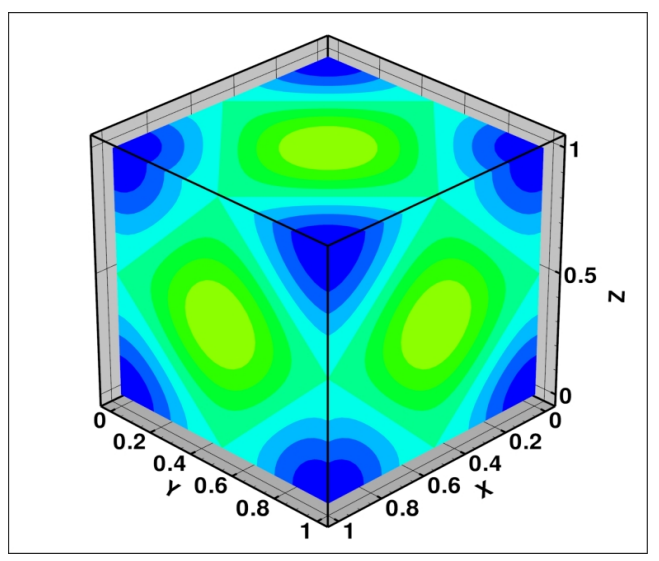

(d) Density profile at $t=3 \mathrm{~T} / 4$

Figure 4: The results are obtained on a $32 \times 32 \times 32$ grid with $\Delta t=T / 20$, where the $T$ is the time period of the standing, compressible waves in a closed cuboid. 
several wave periods. Figure 5 gives an example of the numerical solution during one period. Figure [6] shows that conservation of energy and discrete zero-divergence in time are maintained up to machine precision. To ensure that the velocity field has zero-divergence, one has to initialise the numerical scheme with an exact discrete divergence-free velocity field, see Section 4.3. Thus, adjustment of the initial projection of the velocity field onto the discontinuous Galerkin basis is required to satisfy this condition exactly (up to machine precision). The energy change observed at $t=0$ in Figure 6, originates from this projection, and is within the order of accuracy of the numerical approximation. Table \$ presents the rate of convergence of the Hamiltonian DGFEM discretisation.

Table 3: Convergence of the error in the Hamiltonian DGFEM discretisation for incompressible periodic waves in a cuboid. Due to symmetry all velocity components have the same error.

\begin{tabular}{ll|ll|ll|ll}
\hline \hline & & \multicolumn{2}{|c|}{$p=0$} & & \multicolumn{2}{c|}{$p=1$} & \multicolumn{2}{c}{$p=2$} \\
\hline Grid & & $l^{2}$-error & order & $l^{2}$-error & order & $l^{2}$-error & order \\
\hline $4 \times 4 \times 4$ & $\mathbf{u}$ & $2.665 \mathrm{E}-1$ & - & $1.6340 \mathrm{E}-1$ & - & $7.2122 \mathrm{E}-3$ & - \\
& $p$ & $2.872 \mathrm{E}-2$ & - & $1.3876 \mathrm{E}-2$ & - & $3.9242 \mathrm{E}-3$ & - \\
\hline $8 \times 8 \times 8$ & $\mathbf{u}$ & $1.477 \mathrm{E}-1$ & 0.9 & $5.3412 \mathrm{E}-2$ & 1.6 & $9.6455 \mathrm{E}-4$ & 2.9 \\
& $p$ & $1.411 \mathrm{E}-2$ & 1.0 & $4.6244 \mathrm{E}-3$ & 1.6 & $6.0758 \mathrm{E}-4$ & 2.7 \\
\hline \multirow{2}{*}{$16 \times 16 \times 16$} & $\mathbf{u}$ & $7.587 \mathrm{E}-2$ & 1.0 & $1.8100 \mathrm{E}-2$ & 1.6 & $1.2843 \mathrm{E}-4$ & 2.9 \\
& $p$ & $7.141 \mathrm{E}-3$ & 1.0 & $1.4475 \mathrm{E}-3$ & 1.7 & $1.0251 \mathrm{E}-4$ & 2.6 \\
\hline $32 \times 32 \times 32$ & $\mathbf{u}$ & $3.822 \mathrm{E}-2$ & 1.0 & $5.7218 \mathrm{E}-3$ & 1.7 & $1.6820 \mathrm{E}-5$ & 2.9 \\
& $p$ & $3.737 \mathrm{E}-3$ & 0.9 & $4.5473 \mathrm{E}-4$ & 1.7 & $1.443 \mathrm{E}-5$ & 2.8 \\
\hline $64 \times 64 \times 64$ & $\mathbf{u}$ & $1.919 \mathrm{E}-2$ & 1.0 & $1.6772 \mathrm{E}-3$ & 1.8 & $2.2143 \mathrm{E}-6$ & 2.9 \\
& $p$ & $2.169 \mathrm{E}-3$ & 0.8 & $1.3692 \mathrm{E}-4$ & 1.7 & $2.0682 \mathrm{E}-6$ & 2.8 \\
\hline
\end{tabular}

\subsection{Poincaré waves in a channel}

Poincaré waves in a channel for incompressible flow are considered next. The channel is periodic in the $y$-direction and closed with walls in the $x$-and $z$ - directions. The angular rotation vector is equal to $\Omega=(0,0,1)$. An exact solution for Poincaré waves in $D=[0,1]^{3}$ reads

$$
\begin{aligned}
u & =-\frac{k \sigma^{3}}{1-\sigma^{2}}\left(1+\frac{l^{2}}{(\sigma k)^{2}}\right) \sin (k x) \sin (l y-\sigma t) \cos (2 \pi z), \\
v & =\left(-l \sigma \cos (k x)+\frac{1}{k} \sin (k x)\right) \cos (l y-\sigma t) \cos (2 \pi z), \\
w & =\sigma\left(\cos (k x)+\frac{l}{\sigma k} \sin (k x)\right) \sin (l y-\sigma t) \sin (2 \pi z), \\
P & =-\sigma^{2}\left(\cos (k x)+\frac{l}{\sigma k} \sin (k x)\right) \cos (l y-\sigma t) \cos (2 \pi z),
\end{aligned}
$$




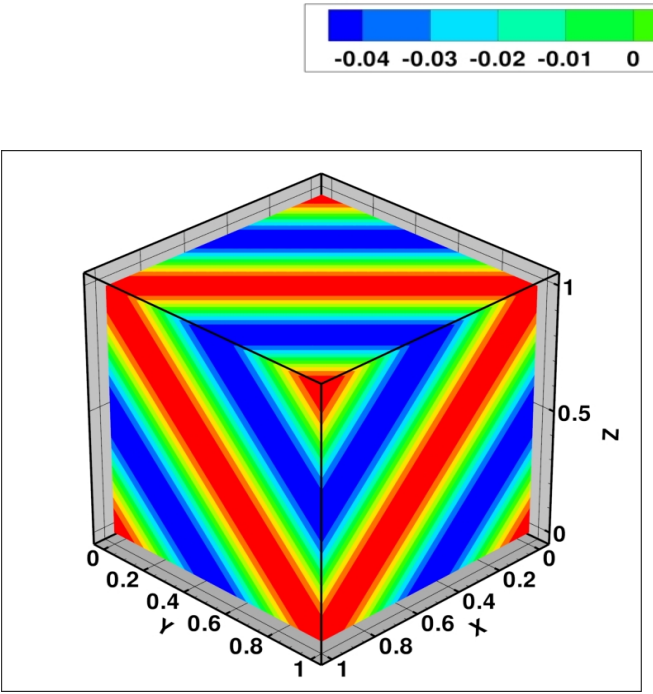

(a) Pressure profile at $\mathrm{t}=0$

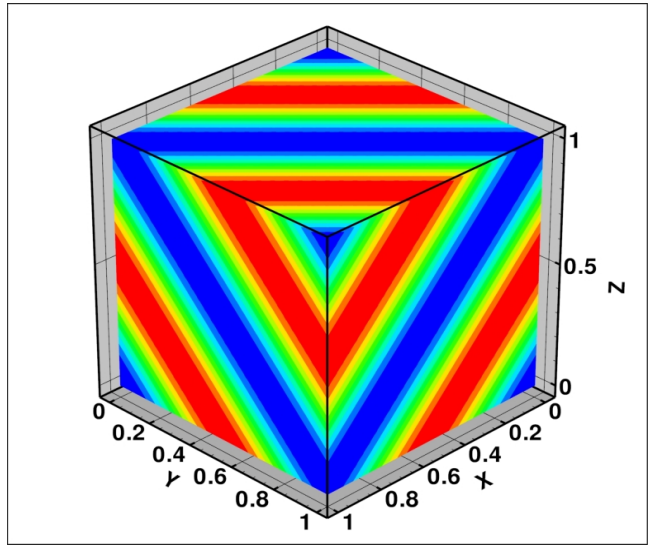

(c) Pressure profile at $\mathrm{t}=\mathrm{T} / 2$

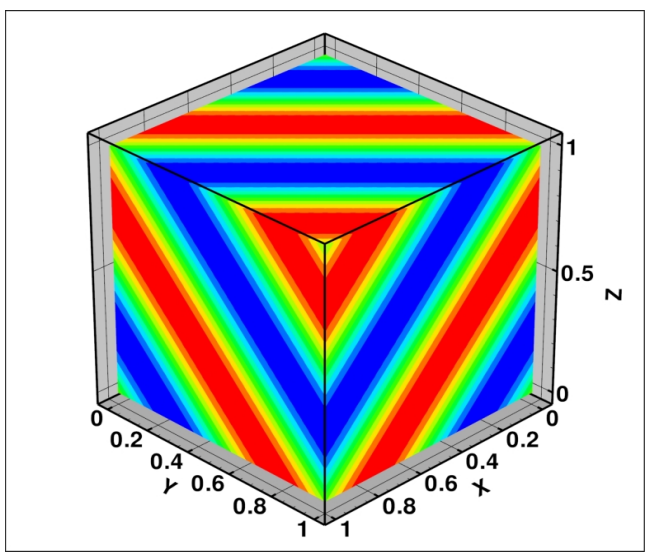

(b) Pressure profile at $\mathrm{t}=\mathrm{T} / 4$

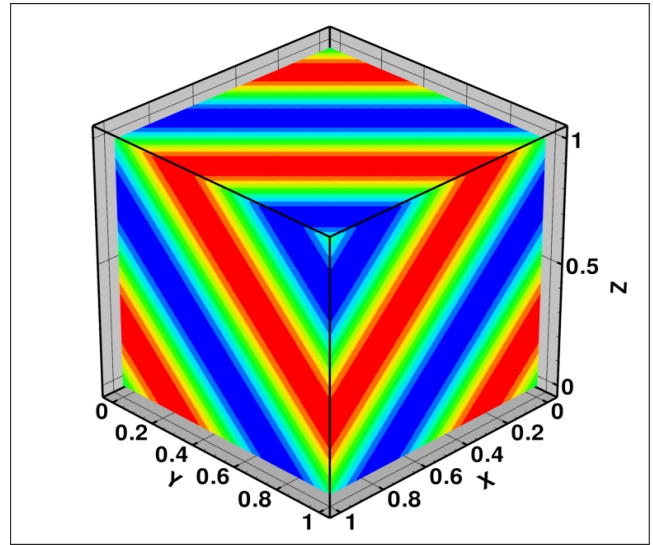

(d) Pressure profile at $\mathrm{t}=3 \mathrm{~T} / 4$

Figure 5: Incompressible waves in periodic domain. The results concern an incompressible Hamiltonian discretisation on a $32 \times 32 \times 32$ grid with $\Delta t=T / 20$ and period $T$. The implementation concerns a quadratic polynomial approximation on local elements. 


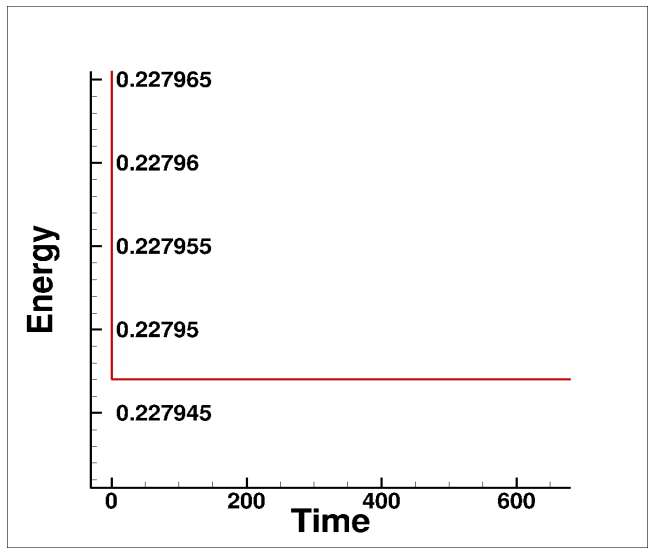

(a) Energy function.

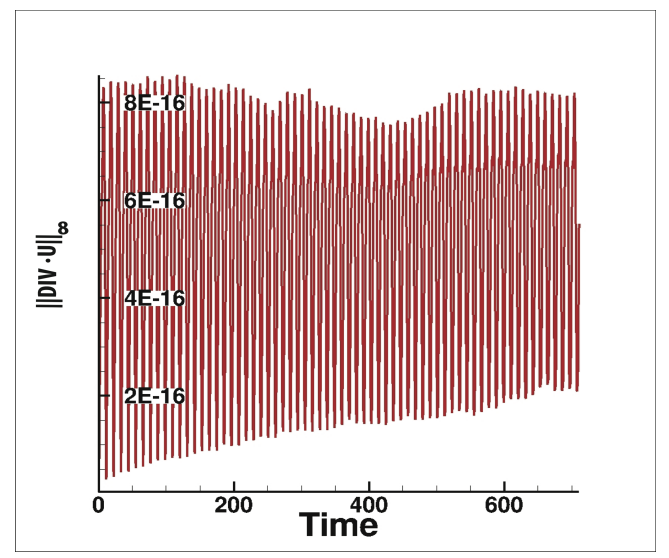

(b) Discrete divergence.

Figure 6: Energy and $L_{\infty}$-norm of discrete divergence in the Hamiltonian DGFEM discretisation during 100 periods of periodic inertial waves in a cuboid.

where $k=2 \pi, l=2 \pi$, and frequency $\sigma=1 / \sqrt{k^{2}+l^{2}+1}$. At the solid walls in the $x$ - and $z$-directions we need to satisfy geostrophic balance at the boundaries, due to the rotation of the domain. In Figures $\square$ and $\nabla$ we present a numerical solution (velocity vector and scalar pressure fields) during one period. Figure 9 demonstrates the discrete conservation of the energy and the zero-divergence of the discrete velocity. Convergence results are given in Table 4 , which show that the convergence rates are close to $k+1$, with $k$ the polynomial order.

\subsection{Inertial waves}

Next, we consider linear, incompressible, rotational fluid flow in a rectangular box with solid wall boundary conditions on all sides. Such kind of flow will lead to inertial waves in the interior of the domain, e.g., [22]. An extensive discussion of these waves as well as an improved semi-analytical solution of this problem can be found in [30]. The semi-analytical solution is used as a test solution for the verification of our incompressible Hamiltonian discretisation with slip-flow boundary conditions. Due to the slow convergence of the semi-analytical solutions, this comparison can, however, only be done for restricted mesh sizes.

Since the exact solution is unknown, we use solutions on a sequence of uniform meshes to obtain an estimate for the rate of convergence, which is called Richardson extrapolation (e.g., [44]). We take a uniformly refined 
Table 4: Convergence of the error in the Hamiltonian DGFEM discretisation for incompressible Poincaré waves in a channel.

\begin{tabular}{ll|ll|ll|ll}
\hline \hline & & \multicolumn{2}{|c|}{$p=0$} & \multicolumn{2}{c|}{$p=1$} & \multicolumn{2}{c}{$p=2$} \\
\hline Grid & & $l^{2}$-error & order & $l^{2}$-error & order & $l^{2}$-error & order \\
\hline $4 \times 4 \times 4$ & $u$ & $7.834 \mathrm{e}+0$ & - & $1.3599 \mathrm{e}+0$ & - & $2.9126 \mathrm{E}-1$ & - \\
& $v$ & $7.492 \mathrm{e}+0$ & - & $1.4793 \mathrm{e}+0$ & - & $2.5108 \mathrm{E}-1$ & - \\
& $w$ & $8.935 \mathrm{e}+0$ & - & $1.6662 \mathrm{e}+0$ & - & $2.4933 \mathrm{E}-1$ & - \\
& $p$ & $5.819 \mathrm{e}+0$ & - & $4.7072 \mathrm{e}+0$ & - & $2.1281 \mathrm{E}-1$ & - \\
\hline $8 \times 8 \times 8$ & $u$ & $3.889 \mathrm{e}+0$ & 1.0 & $5.7839 \mathrm{E}-1$ & 1.3 & $2.8640 \mathrm{E}-2$ & 3.3 \\
& $v$ & $3.802 \mathrm{e}+0$ & 1.0 & $6.1504 \mathrm{E}-1$ & 1.3 & $2.0699 \mathrm{E}-2$ & 3.6 \\
& $w$ & $4.042 \mathrm{e}+0$ & 1.1 & $6.7238 \mathrm{E}-1$ & 1.3 & $2.0075 \mathrm{E}-2$ & 3.6 \\
& $p$ & $2.290 \mathrm{e}+0$ & 1.3 & $9.1548 \mathrm{E}-1$ & 2.3 & $2.9025 \mathrm{E}-2$ & 2.9 \\
\hline $16 \times 16 \times 16$ & $u$ & $2.192 \mathrm{e}+0$ & 0.8 & $1.9858 \mathrm{E}-1$ & 1.5 & $3.1792 \mathrm{E}-3$ & 3.1 \\
& $v$ & $2.229 \mathrm{e}+0$ & 0.8 & $2.4017 \mathrm{E}-1$ & 1.4 & $2.3963 \mathrm{E}-3$ & 3.1 \\
& $w$ & $2.015 \mathrm{e}+0$ & 1.0 & $2.4244 \mathrm{E}-1$ & 1.5 & $2.2733 \mathrm{E}-3$ & 3.1 \\
& $p$ & $1.179 \mathrm{e}+0$ & 1.0 & $3.2671 \mathrm{E}-1$ & 1.5 & $3.2460 \mathrm{E}-3$ & 3.1 \\
\hline $32 \times 32 \times 32$ & $u$ & $1.136 \mathrm{e}+0$ & 0.9 & $6.3126 \mathrm{E}-2$ & 1.6 & $4.1469 \mathrm{E}-4$ & 2.9 \\
& $v$ & $1.169 \mathrm{e}+0$ & 0.9 & $8.4394 \mathrm{E}-2$ & 1.5 & $3.2670 \mathrm{E}-4$ & 2.9 \\
& $w$ & $1.065 \mathrm{e}+0$ & 0.9 & $8.5931 \mathrm{E}-2$ & 1.5 & $3.1967 \mathrm{E}-4$ & 2.8 \\
& $p$ & $5.932 \mathrm{E}-1$ & 1.0 & $1.0317 \mathrm{E}-1$ & 1.7 & $4.3572 \mathrm{E}-4$ & 2.9 \\
\hline $64 \times 64 \times 64$ & $u$ & $5.726 \mathrm{E}-1$ & 1.0 & $1.8031 \mathrm{E}-2$ & 1.8 & $5.5452 \mathrm{E}-5$ & 2.9 \\
& $v$ & $5.899 \mathrm{E}-1$ & 1.0 & $2.4461 \mathrm{E}-2$ & 1.8 & $4.4548 \mathrm{E}-5$ & 2.9 \\
& $w$ & $5.258 \mathrm{E}-1$ & 1.0 & $2.3687 \mathrm{E}-2$ & 1.9 & $4.3963 \mathrm{E}-5$ & 2.9 \\
& $p$ & $2.961 \mathrm{E}-1$ & 1.0 & $3.1627 \mathrm{E}-2$ & 1.7 & $4.3572 \mathrm{E}-5$ & 2.9 \\
\hline
\end{tabular}




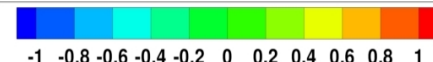

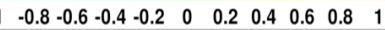

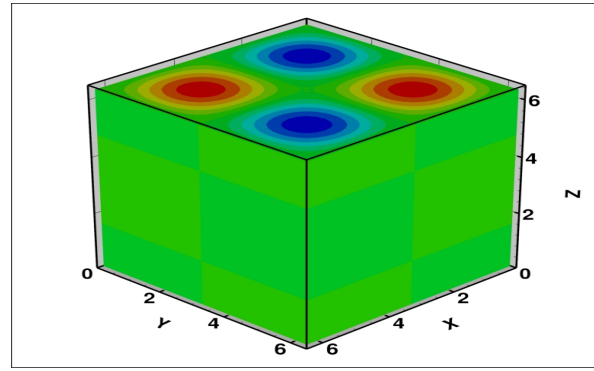

(a) $u$ component at $\mathrm{t}=0$

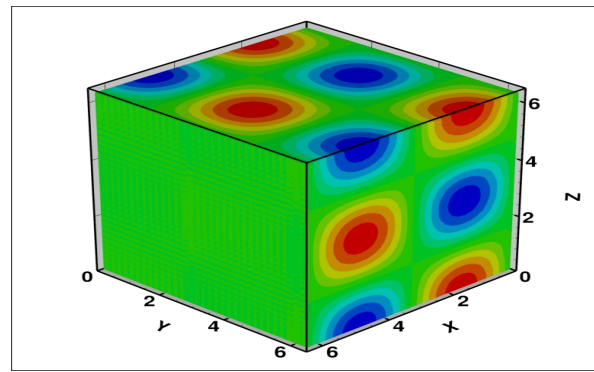

(c) $u$ component at $\mathrm{t}=\mathrm{T} / 4$

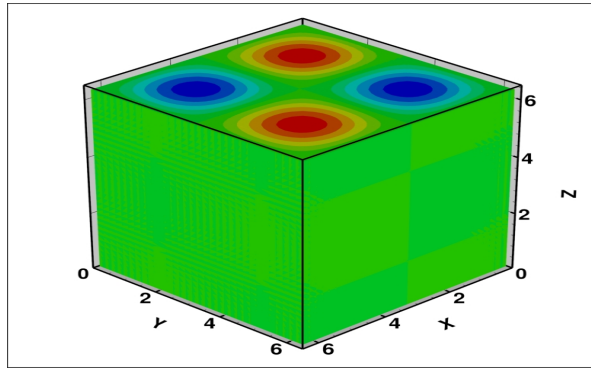

(e) $u$ component at $\mathrm{t}=\mathrm{T} / 2$

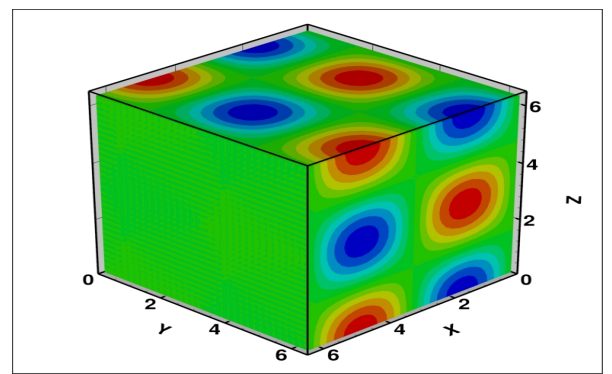

(g) $u$ component at $\mathrm{t}=3 \mathrm{~T} / 4$

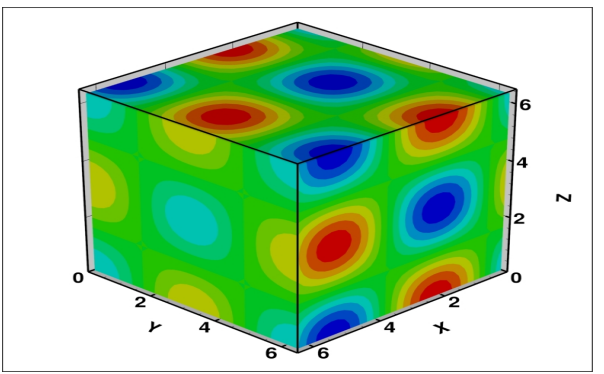

(b) $v$ component at $\mathrm{t}=0$

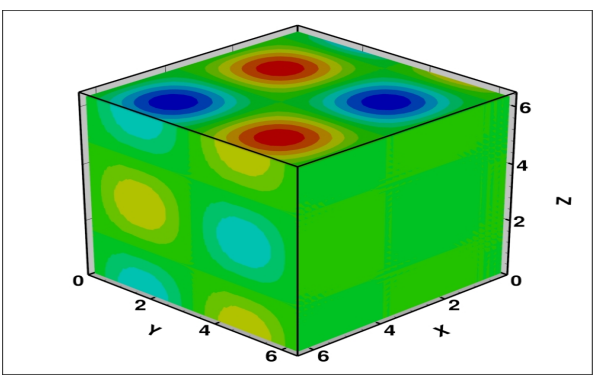

(d) $v$ component at $\mathrm{t}=\mathrm{T} / 4$

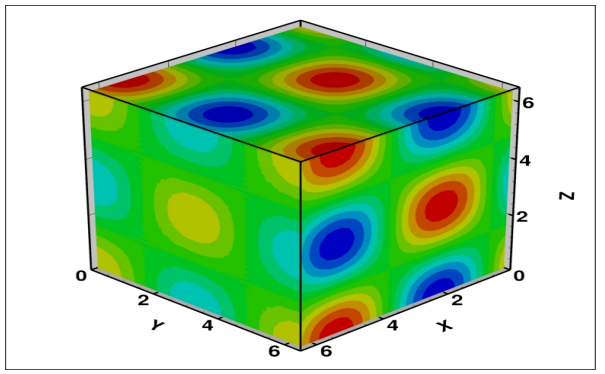

(f) $v$ component at $\mathrm{t}=\mathrm{T} / 2$

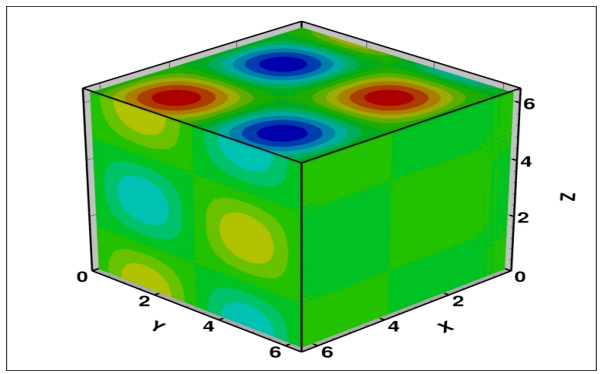

(h) $v$ component at $\mathrm{t}=3 \mathrm{~T} / 4$

Figure 7: Velocity components $u$ and $v$ for Poincaré waves are computed. Numerical results concern the incompressible Hamiltonian DGFEM discretisation on a $32 \times 32 \times 32$ grid with $\Delta t=T / 20$. We consider a quadratic polynomial approximation in local elements. 


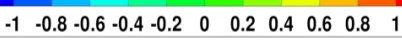

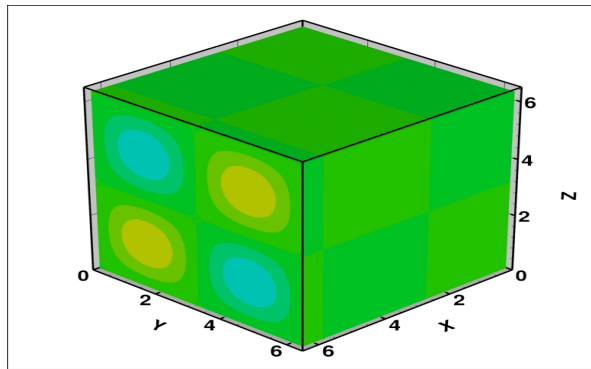

(a) $w$ component at $\mathrm{t}=0$

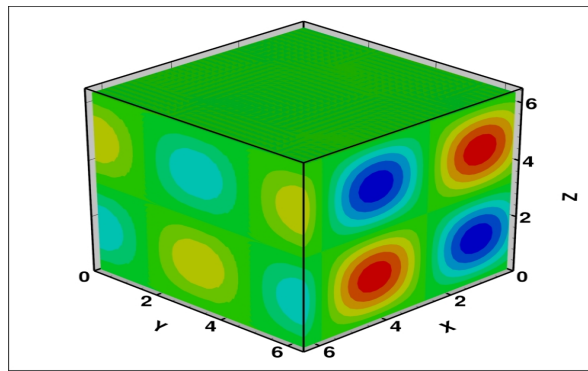

(c) $w$ component at $\mathrm{t}=\mathrm{T} / 4$

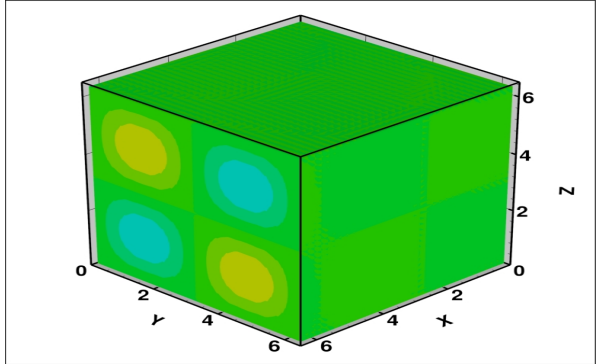

(e) $w$ component at $\mathrm{t}=\mathrm{T} / 2$

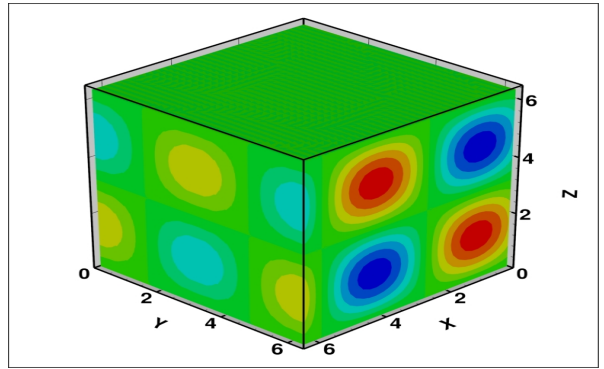

(g) $w$ component at $\mathrm{t}=3 \mathrm{~T} / 4$

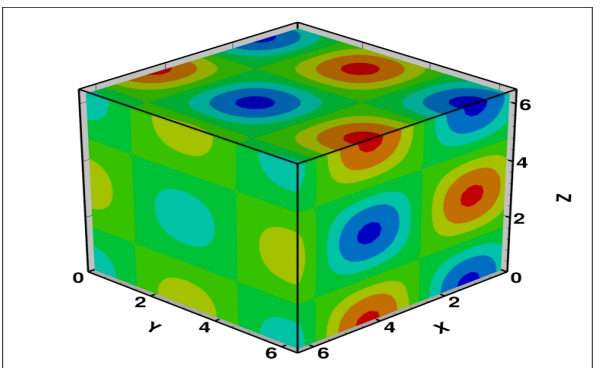

(b) $p$ scalar pressure at $\mathrm{t}=0$

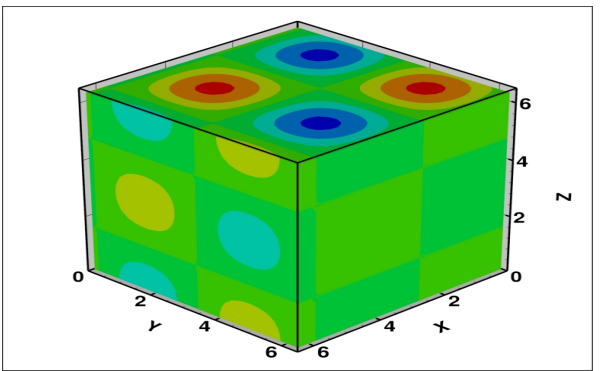

(d) $p$ scalar pressure at $\mathrm{t}=\mathrm{T} / 4$

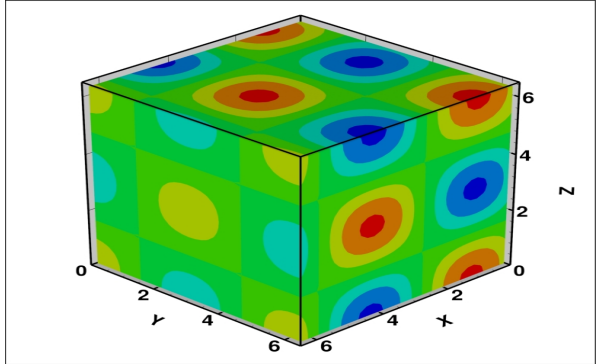

(f) $p$ scalar pressure at $\mathrm{t}=\mathrm{T} / 2$

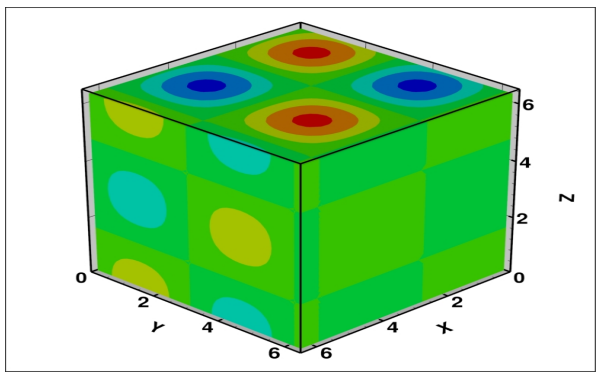

(h) $p$ scalar pressure at $\mathrm{t}=3 \mathrm{~T} / 4$

Figure 8: Vertical velocity component $w$ and linearised scalar pressure fields during one period of a Poincaré-wave simulation. For details, see the caption of Figure $\square$. 


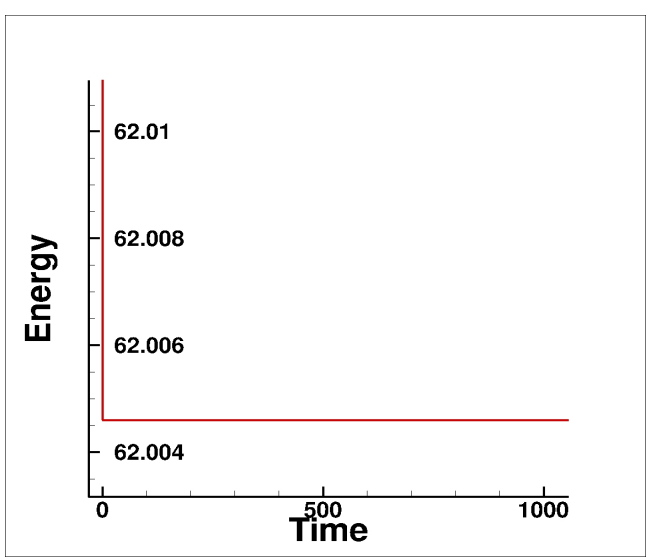

(a) Energy function.

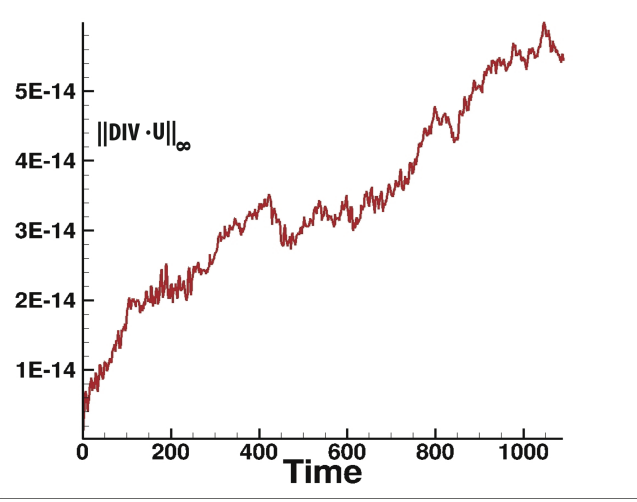

(b) Discrete divergence.

Figure 9: Energy and $L_{\infty}$-norm of discrete divergence-free velocity field during 100 periods in Hamiltonian DGFEM computations of a Poincaré-wave.

sequence of meshes $h_{4}=h_{\text {ref }}<h_{3}<h_{2}<h_{1}$, where the mesh-size $h_{i}$ $(i=1,2,3.4)$ is doubled for each finer mesh, and calculate the convergence rate $s$ by numerically solving the following equation

$$
\frac{h_{1}^{s}-h_{2}^{s}}{h_{2}^{s}-h_{3}^{s}}=\frac{\left\|\mathbf{U}_{\mathrm{ref}}-\mathbf{U}_{h_{1}}\right\|-\left\|\mathbf{U}_{\mathrm{ref}}-\mathbf{U}_{h_{2}}\right\|}{\left\|\mathbf{U}_{\mathrm{ref}}-\mathbf{U}_{h_{2}}\right\|-\left\|\mathbf{U}_{\mathrm{ref}}-\mathbf{U}_{h_{3}}\right\|} .
$$

The numerical velocity field and the mesh size for the different meshes are given with subscript notation, where $(\cdot)_{\text {ref }}$ denotes the finest mesh. By taking the sequence of meshes $64 \times 64 \times 64,32 \times 32 \times 32,16 \times 16 \times 16,8 \times 8 \times 8$, we numerically solve ( 82$)$. The convergence rate in the $L_{\infty}$-norm is roughly as expected, $s \approx 2.89$, for the implementation with quadratic polynomials.

The extensive tests reported above convince us that the presented numerical scheme is actually more accurate than the slowly converging semi-

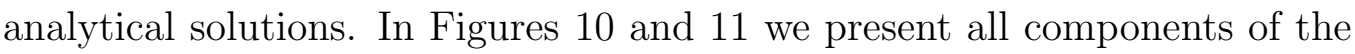
numerical velocity vector and pressure fields produced by a simulation of incompressible fluid flow initialised with one of the eigenmodes of the semianalytical solution. The domain is a rectangular box $D=[0,2 \pi] \times[0, \pi]^{2}$. Figure [2 shows conservation of energy and discrete zero-divergence. 


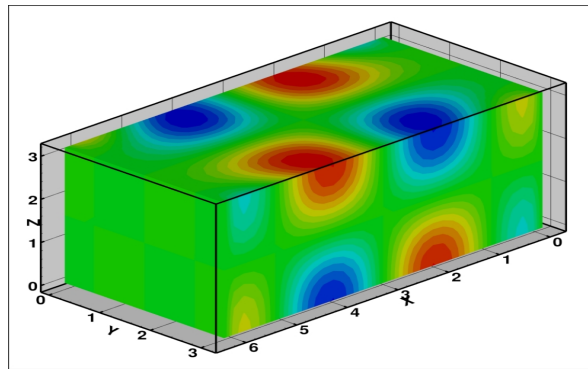

(a) $u$ component at $\mathrm{t}=0$

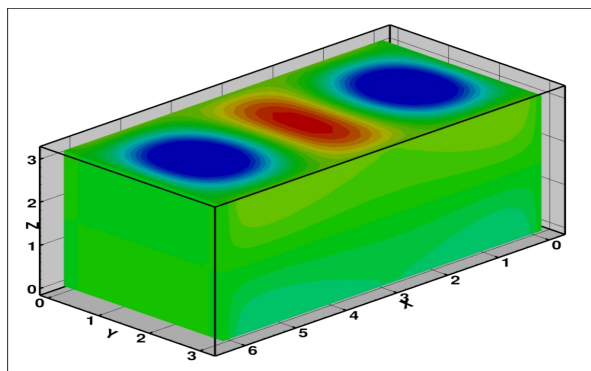

(c) $u$ component at $\mathrm{t}=\mathrm{T} / 4$

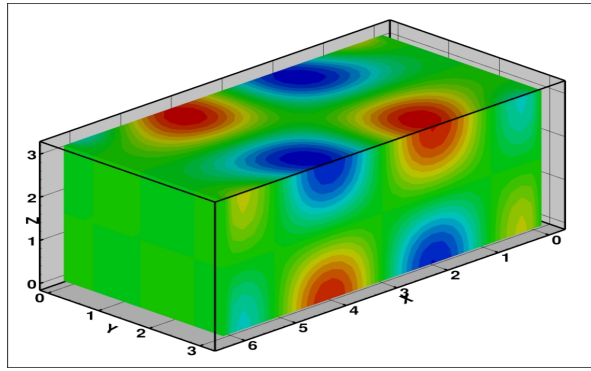

(e) $u$ component at $\mathrm{t}=\mathrm{T} / 2$

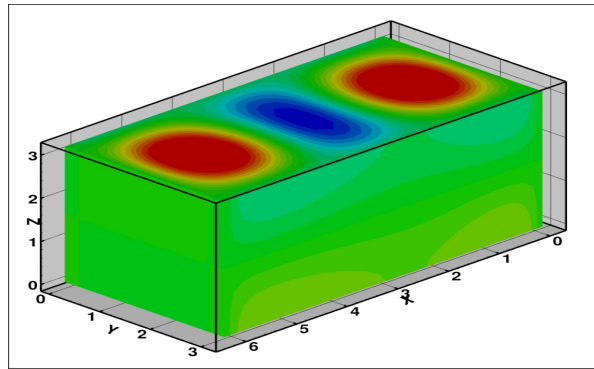

(g) $u$ component at $\mathrm{t}=3 \mathrm{~T} / 4$

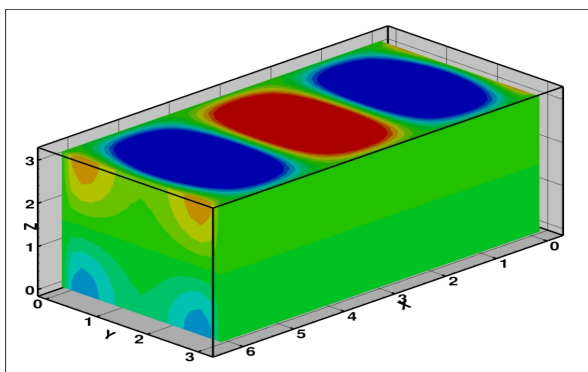

(b) $v$ component at $\mathrm{t}=0$

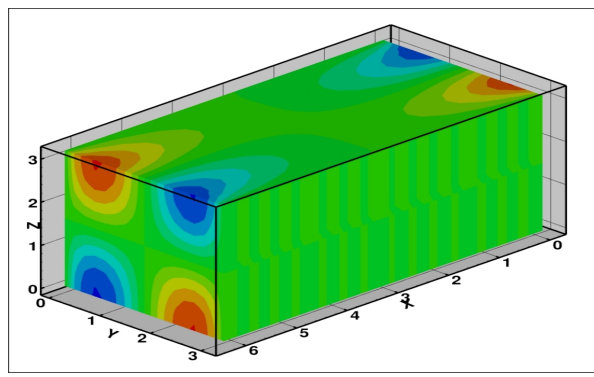

(d) $v$ component at $\mathrm{t}=\mathrm{T} / 4$

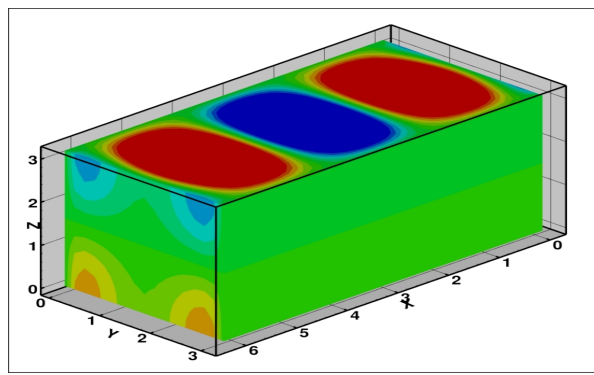

(f) $v$ component at $\mathrm{t}=\mathrm{T} / 2$

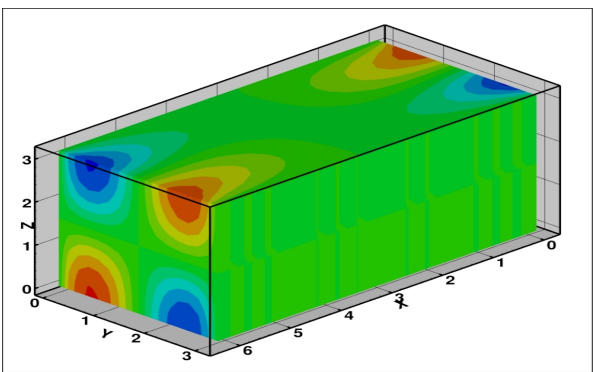

(h) $v$ component at $\mathrm{t}=3 \mathrm{~T} / 4$

Figure 10: Horizontal velocity components $u$ and $v$ of an inertial wave with eigenfrequency $\sigma=0.477$. The rotation vector is aligned with the $z$-direction. Quadratic basis functions are used on $32 \times 16 \times 16$ mesh with time step $\Delta t=T / 20$. 


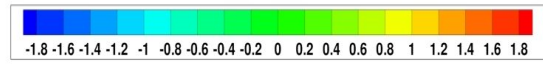

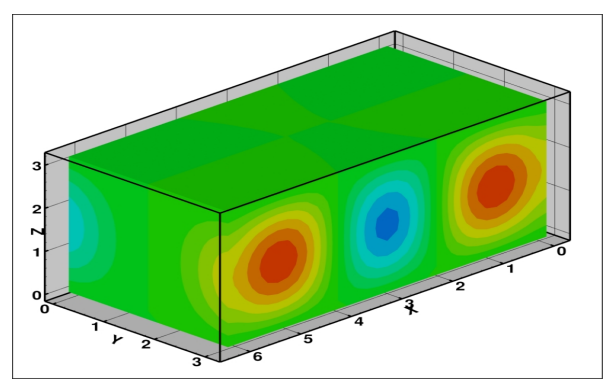

(a) $w$ component at $\mathrm{t}=0$

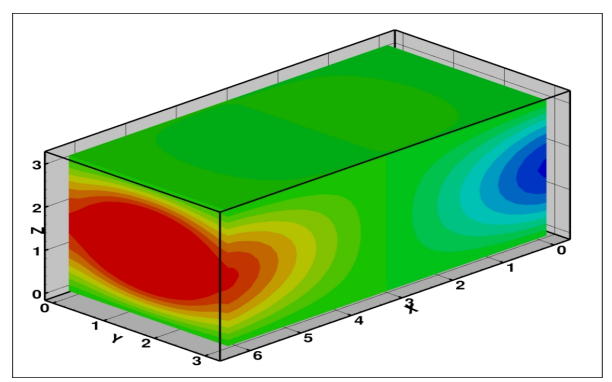

(c) $w$ component at $\mathrm{t}=\mathrm{T} / 4$

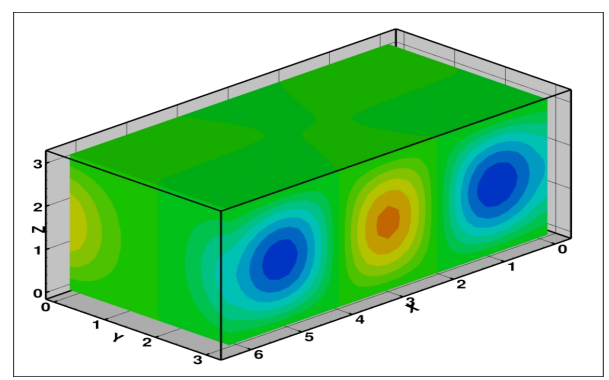

(e) $w$ component at $\mathrm{t}=\mathrm{T} / 2$

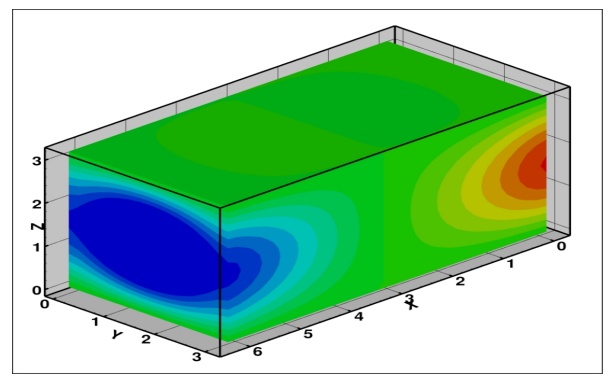

(g) $w$ component at $\mathrm{t}=3 \mathrm{~T} / 4$

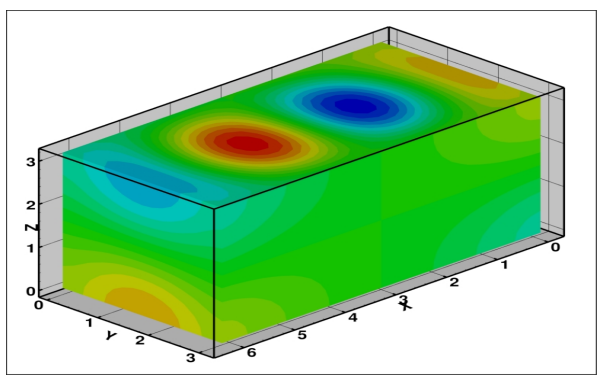

(b) $p$ scalar pressure at $\mathrm{t}=0$

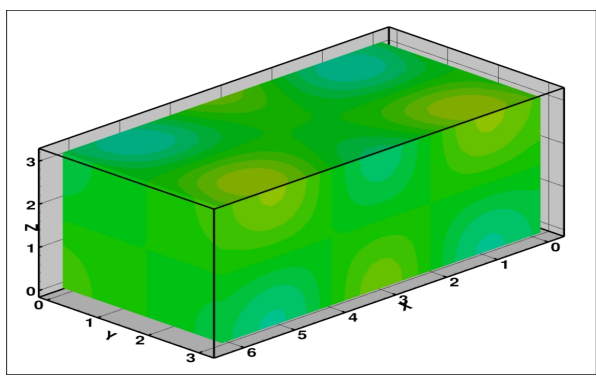

(d) $p$ scalar pressure at $\mathrm{t}=\mathrm{T} / 4$

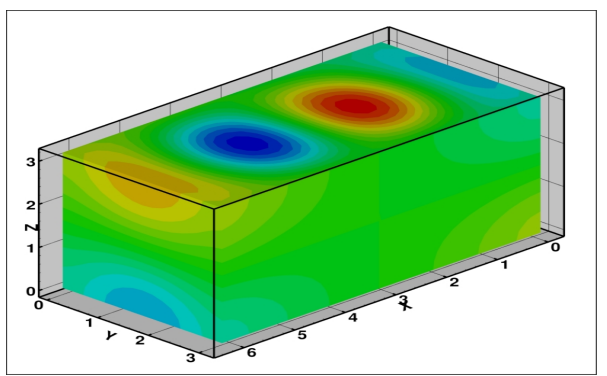

(f) $p$ scalar pressure at $\mathrm{t}=\mathrm{T} / 2$

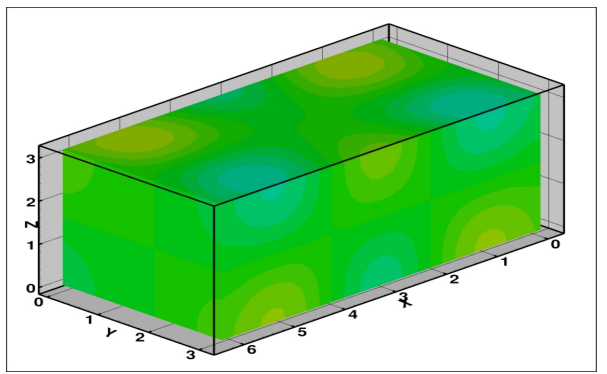

(h) $p$ scalar pressure at $\mathrm{t}=3 \mathrm{~T} / 4$

Figure 11: Vertical component $w$ of velocity and scalar pressure $p$ for the inertial wave simulation. For details, see the caption of Figure 皿. 


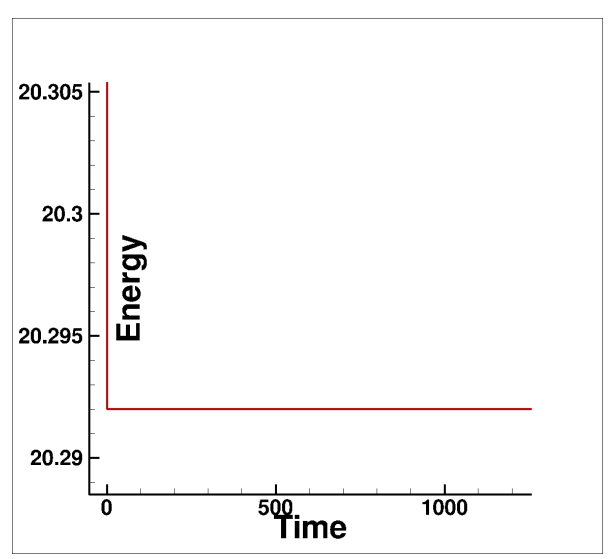

(a) Energy function.

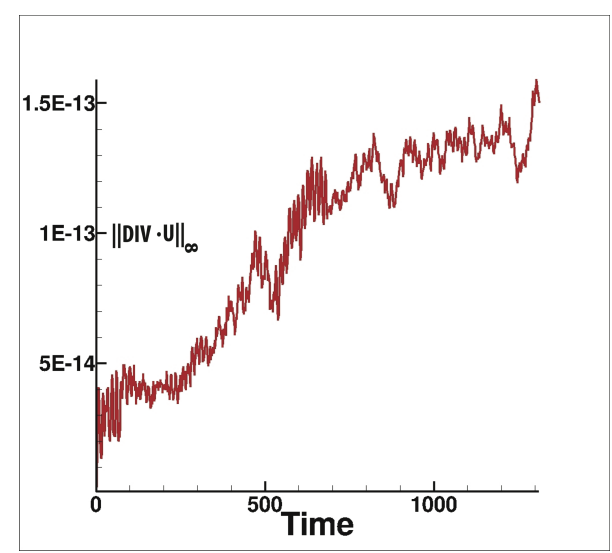

(b) Discrete divergence.

Figure 12: Energy and $L_{\infty}$-norm of discrete divergence-free velocity in Hamiltonian DGFEM discretisation during 100 time periods of inertial waves in a cuboid.

\subsection{Inertial waves in a 'tilted' box}

Finally, we attempt to observe wave focussing in our numerical wave tank, to demonstrate the capabilities of our novel numerical scheme. In the previous cases, the walls are either parallel or perpendicular to the rotation vector, possessing a "local reflectional symmetry". Thus no mode breaking can be observed. However, a slight tilt in one of the walls results in symmetry breaking and hence in wave focussing and defocussing, such that, due to dominance of the former, wave attractors may appear [25]. Here we break the "local reflectional symmetry" by a small change in the background angular velocity vector.

The domain is chosen to be a prolonged three-dimensional box with $D=[0,4 \pi] \times[0, \pi]^{2}$. The simulation is initialised with a particular eigenfrequency $(\sigma \approx 0.6946)$ from the semi-analytical solution for a domain with a constant background rotation $\left(\Omega_{*}=(0,0,1)\right)$ aligned along the $z$-direction available from [25, [30]. Next we introduce a small tilt in the angular velocity such that $\Omega=(0,0.1,1)$. After some time, the initial mode completely changes its structure and its frequency, due to the 'tilt'; see the plots of the 'tilted' and original flows in Figures [.3] and [4]. The distribution of the energy in the 'tilted' rectangular domain is given in Figure ㄷ.5. In the vertical cross-section of the energy distribution plot (see Figure [5), a structure rem- 
iniscent of a wave attractor can be discerned. This rectangular region with a locally increased energy density is most evident in the middle of the tank, whereas in the rest of the tank there is a lower energy distribution. Furthermore, a similar rectangular structure can be seen in the pressure field, whereas the pressure field of the original 'untilted' mode has a regular structure (see Figures 103 and [4). The numerical solution is expanded in terms of quadratic polynomials defined on a mesh with $160 \times 40 \times 40$ elements. In the current implementation the size of the mesh is constrained by the available random-access memory. The latter obstacle can be overcome by applying local $h p$-refinement near the zones with high wave amplitude and implementing the numerical algorithm in a parallel environment.

\section{Concluding remarks}

We have derived a DGFEM discretisation for Hamiltonian dynamics of linear, rotating incompressible fluid flow. The discretisation was obtained by applying Dirac's constrained Hamiltonian theory on a DGFEM formulation of compressible fluid flows. As an interim result a discretisation of Hamiltonian dynamics of compressible flow was derived, implemented and tested against exact solutions. The use of Dirac's theory is a novel approach to derive a Hamiltonian discontinuous Galerkin discretisation for incompressible flow using a related discretisation for compressible flow. The resulting system, as a consequence of the exact preservation of the constraints, does not require a stabilisation common to some direct DGFEM discretisations of incompressible fluid flows.

It was a challenge to derive and implement the boundary conditions for a discretisation preserving the Hamiltonian structure in a rotating frame, due to the mandatory satisfaction of geostrophic balance for the flow along fixed walls. Moreover, for exact preservation of energy and zero-divergence, the presented numerical scheme requires the projection of the initial velocity profile to be exactly divergence free at the discrete level. A preprocessing step was thus introduced to ensure that the initial velocity field in the DGFEM discretisation is divergence free up to machine precision.

Several tests of inertial waves in rotating domains were presented. The simulation of Poincaré inertial waves in a channel assessed the proper implementation of no-normal flow boundary conditions in rotating domains. Next, an inertial-wave simulation in a cuboid with fixed solid walls showed agreement up to $10^{-2}$ with slowly converging semi-analytical solutions avail- 


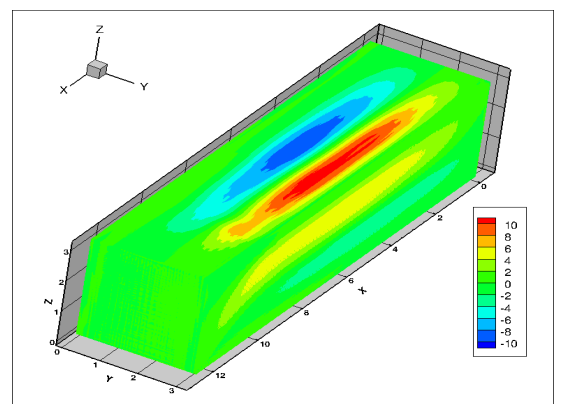

(a) $u$ component

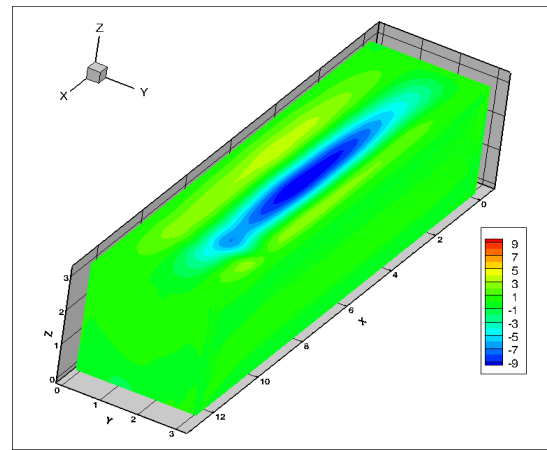

(c) $v$ component

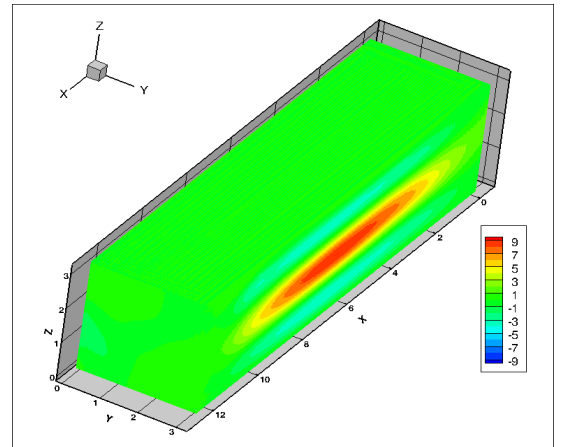

(e) $w$ component

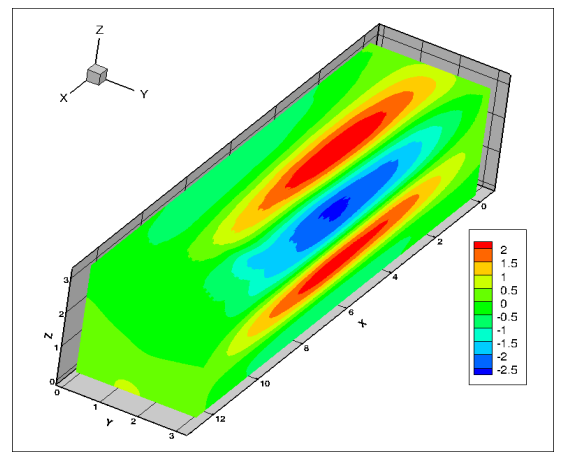

(g) $p$ pressure

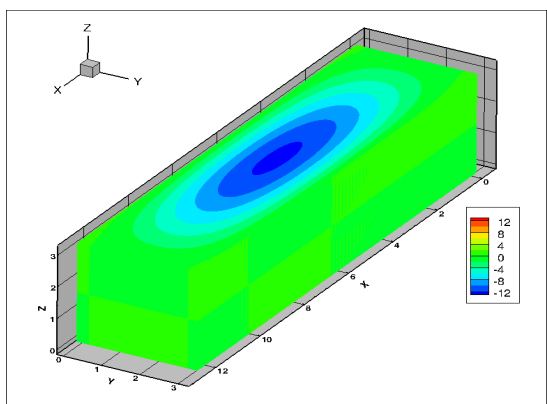

(b) $u$ component of exact mode

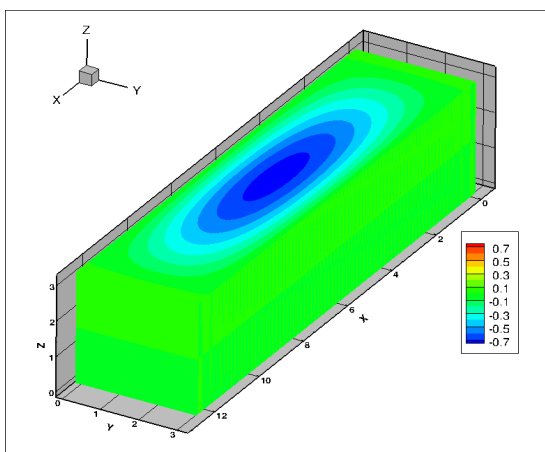

(d) $v$ component of exact mode

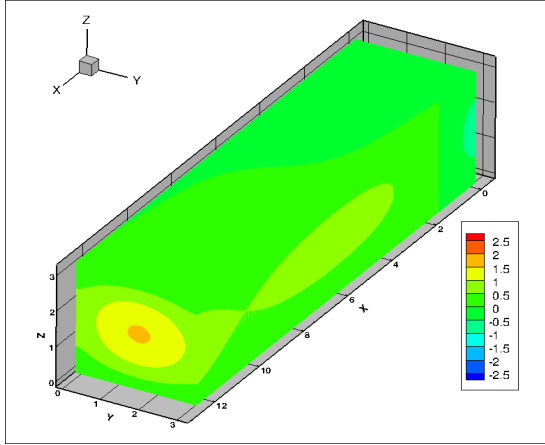

(f) $w$ component of exact mode

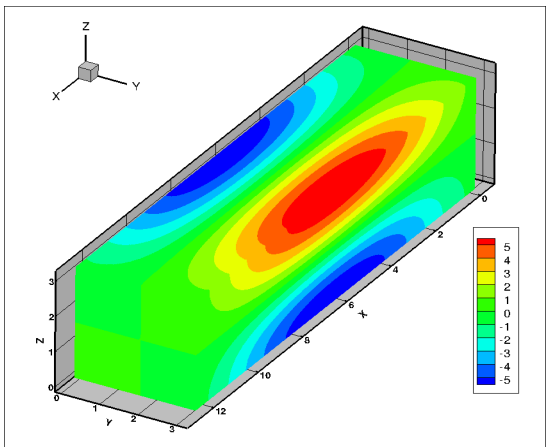

(h) $p$ pressure of exact mode

Figure 13: All components of the velocity field and the pressure field are given at time $\mathrm{t}=56$.4. The first column concerns the 'tilted' simulation and the second column is the exact 'untilted' semi-analytical solution. 


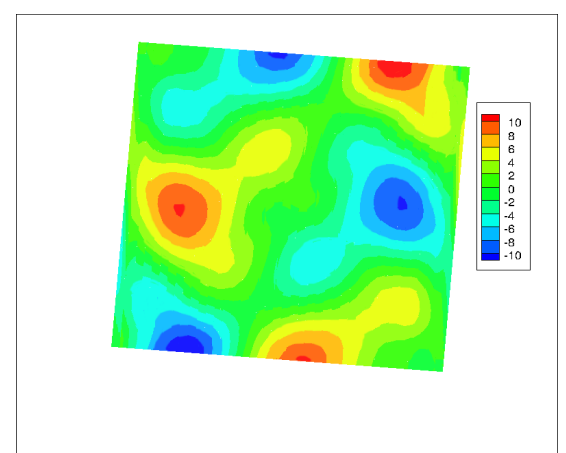

(a) $u$ component

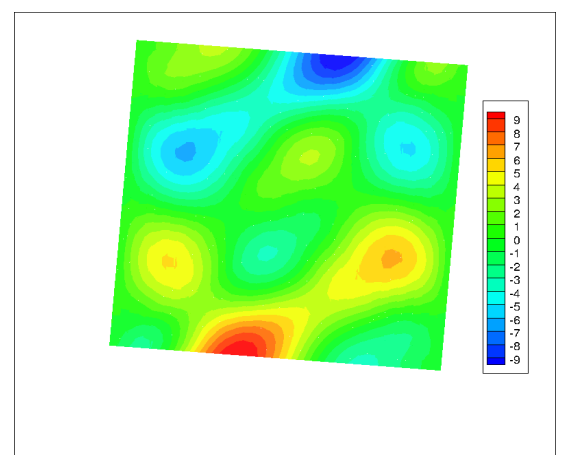

(c) $v$ component

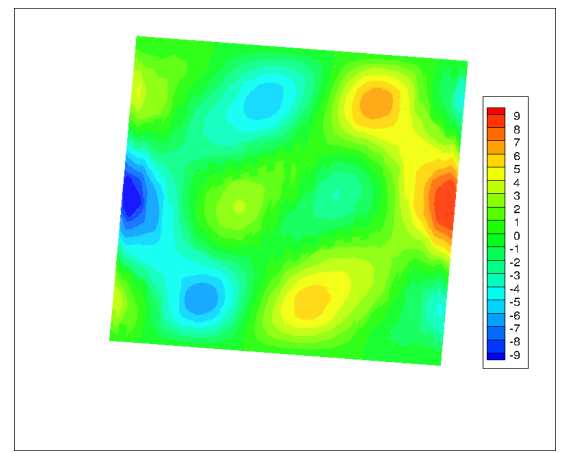

(e) $w$ component

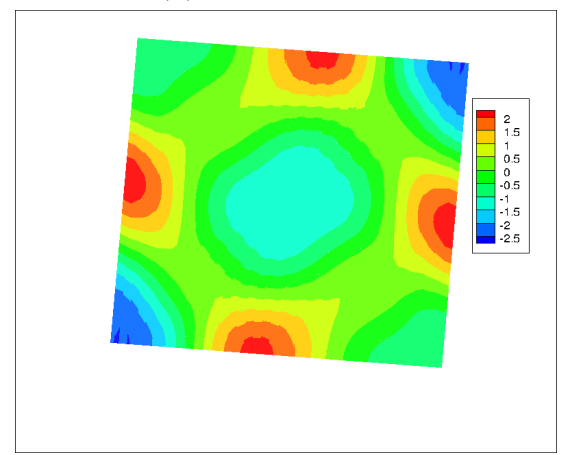

(g) $p$ component

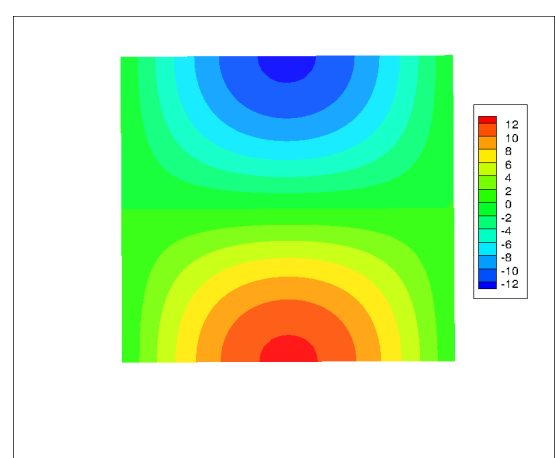

(b) $u$ component of the exact mode

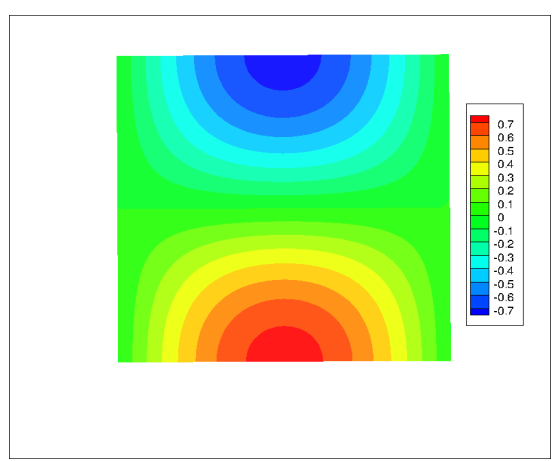

(d) $v$ component of the exact mode

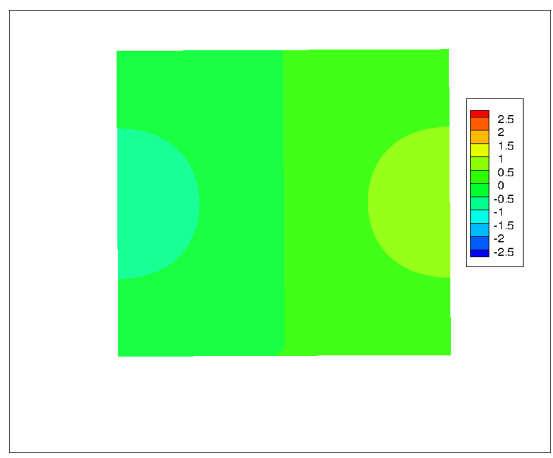

(f) $w$ component of the exact mode

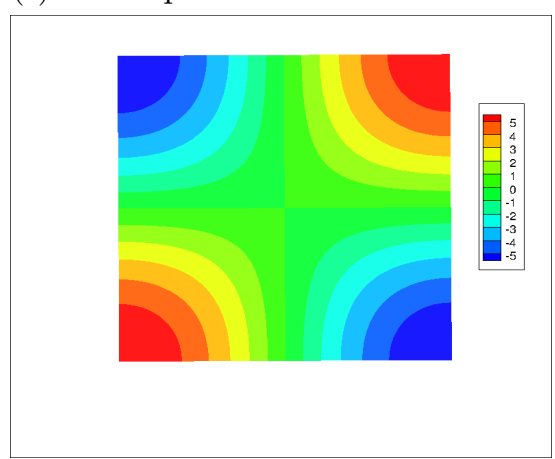

(h) $p$ pressure of the exact mode

Figure 14: Vertical cross-sections of the fields given in Figure [3.3 in the middle of the tank. 


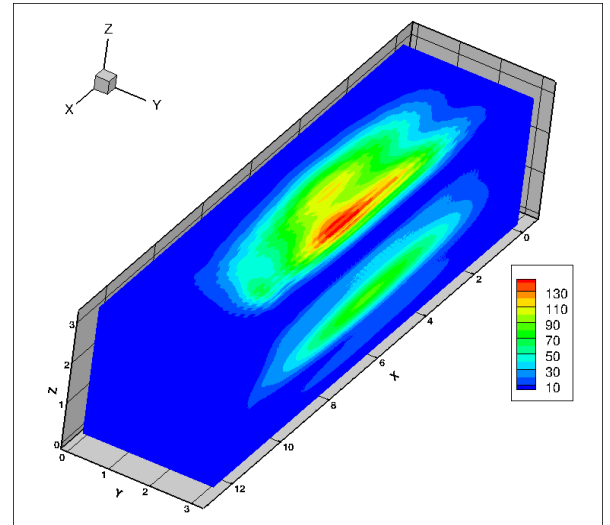

(a) Three-dimensional energy plot.

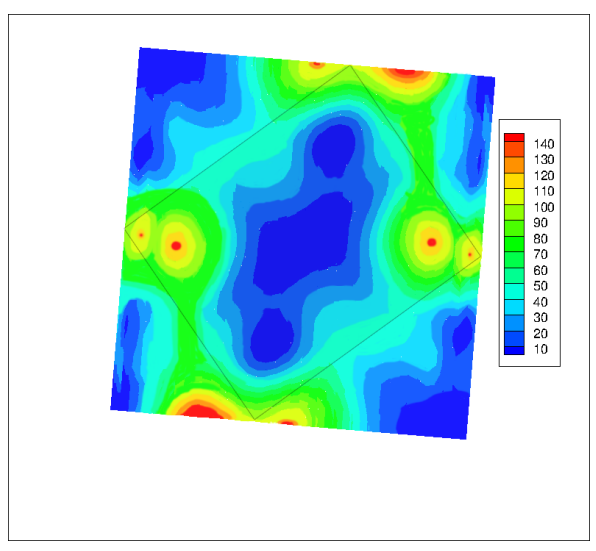

(b) Vertical cross-section in the middle.

Figure 15: The distribution of the numerical energy in the 'tilted' simulation at time $\mathrm{t}=56.4$. In (b), we outlined the tentative attractor.

able from [22]. Richardson extrapolation with sequencing of meshes proved that our numerical solution is more accurate: the semi-analytical solutions available from [22] or [30] do either not satisfy the Euler equations or the solid-wall boundary conditions exactly. A DGFEM allows relatively easy $h p$-refinement of the system. Global $p$-refinement was already used in all presented numerical test cases and it appears that the quadratic polynomial approximation in the local elements provides sufficient order of accuracy for capturing the phenomena of inertial waves in rotating domains.

Moreover, a simulation of inertial waves in a rotating domain with a small change in the direction and magnitude of the background angular velocity revealed a structure reminiscent of a wave attractor, due to the violation of the "local reflectional symmetry". To capture sufficient details of wave attractors, it is useful to introduce $h p$-refinement of the domain near the zones of attraction. This is one of the nice features of a DG scheme and will be addressed in future research. It will also allow more detailed studies of the wave focussing and defocussing in a container deprived of "local reflectional symmetry". Additional work will include the incorporation of a free surface in the presented numerical scheme for incompressible fluid flows. 
Appendix .1. Constrained Hamiltonian continuum dynamics

The first calculation concerns the derivation from (20a) to (स])

$$
\begin{aligned}
0 & =\{\mathcal{F}[\rho], \mathcal{H}\}+\int_{D} \lambda_{\Delta}\left(\mathbf{x}^{\prime}\right)\left\{\mathcal{F}[\rho], \Delta\left(\mathbf{x}^{\prime}\right)\right\} d \mathbf{x}^{\prime} \\
& =-\int_{D} \frac{\delta \mathcal{F}}{\delta \rho} \nabla \cdot\left(\rho_{0} \mathbf{u}\right) d \mathbf{x}-\iint_{D, D^{\prime}} \lambda_{\Delta}(\mathbf{x}) \frac{\delta \mathcal{F}}{\delta \rho\left(\mathbf{x}^{\prime}\right)} \nabla^{\prime} \cdot \frac{\delta \nabla \cdot \mathbf{u}(\mathbf{x})}{\delta \mathbf{u}\left(\mathbf{x}^{\prime}\right)} d \mathbf{x} d \mathbf{x}^{\prime}
\end{aligned}
$$

The last term in (ㅁ) can be reworked to

$$
\begin{aligned}
& -\iint_{D, D^{\prime}} \lambda_{\Delta}(\mathbf{x}) \frac{\delta \mathcal{F}}{\delta \rho\left(\mathbf{x}^{\prime}\right)} \nabla^{\prime} \cdot \frac{\delta \nabla \cdot \mathbf{u}(\mathbf{x})}{\delta \mathbf{u}\left(\mathbf{x}^{\prime}\right)} d \mathbf{x} d \mathbf{x}^{\prime} \\
& =\iint_{D, D^{\prime}} \lambda_{\Delta}(\mathbf{x}) \nabla^{\prime} \frac{\delta \mathcal{F}}{\delta \rho\left(\mathbf{x}^{\prime}\right)} \cdot \frac{\delta \nabla \cdot \mathbf{u}(\mathbf{x})}{\delta \mathbf{u}\left(\mathbf{x}^{\prime}\right)} d \mathbf{x} d \mathbf{x}^{\prime} \\
& \quad-\int_{D} \int_{\partial D^{\prime}} \lambda_{\Delta}(\mathbf{x}) \frac{\delta \mathcal{F}}{\delta \rho\left(\mathbf{x}^{\prime}\right)} \hat{\mathbf{n}}^{\prime} \cdot \frac{\delta \nabla \cdot \mathbf{u}(\mathbf{x})}{\delta \mathbf{u}\left(\mathbf{x}^{\prime}\right)} d S^{\prime} d \mathbf{x} \\
& =-\int_{D} \nabla \lambda_{\Delta} \cdot \nabla \frac{\delta \mathcal{F}}{\delta \rho} d \mathbf{x}+\int_{\partial D} \int_{D^{\prime}} \lambda_{\Delta}(\mathbf{x}) \nabla^{\prime} \frac{\delta \mathcal{F}}{\delta \rho\left(\mathbf{x}^{\prime}\right)} \hat{\mathbf{n}} \cdot \frac{\delta \mathbf{u}(\mathbf{x})}{\delta \mathbf{u}\left(\mathbf{x}^{\prime}\right)} d S d \mathbf{x}^{\prime} \\
& =\int_{D} \frac{\delta \mathcal{F}}{\delta \rho} \nabla^{2} \lambda_{\Delta} d \mathbf{x}-\int_{\partial D} \frac{\delta \mathcal{F}}{\delta \rho} \nabla \lambda_{\Delta} \cdot \hat{\mathbf{n}} d S,
\end{aligned}
$$

in which the first boundary term emerging is zero, and similarly the second boundary term emerging is zero, because

$$
n_{i}^{\prime} \partial_{j}\left(\frac{\delta u_{j}(\mathbf{x})}{\delta u_{i}\left(\mathbf{x}^{\prime}\right)}\right)=\delta_{i j} \partial_{i}\left(n_{i}^{\prime} \frac{\delta u_{i}\left(\mathbf{x}^{\prime}\right)}{\delta u_{i}(\mathbf{x})}\right)=0,
$$

since $\delta u_{i}(\mathbf{x}) / \delta u_{i}\left(\mathbf{x}^{\prime}\right)=\delta u_{i}\left(\mathbf{x}^{\prime}\right) / \delta u_{i}(\mathbf{x})=\delta\left(\mathbf{x}-\mathbf{x}^{\prime}\right)$, which follows by analysing $\delta u_{i}(\mathbf{x})$ and $\delta u_{i}\left(\mathbf{x}^{\prime}\right)$ as functionals, and $n_{i} \delta u_{i}=\delta\left(n_{i} u_{i}\right)=0$ at $\partial D$. The index $i$ denotes the velocity component and summation over repeated indices is understood. Recombining the above yields the result stated in the main text

$$
0=\int_{D} \frac{\delta \mathcal{F}}{\delta \rho}\left(-\nabla \cdot\left(\rho_{0} \mathbf{u}\right)+\nabla^{2} \lambda_{\Delta}\right) d \mathbf{x}-\int_{\partial D} \frac{\delta \mathcal{F}}{\delta \rho} \hat{\mathbf{n}} \cdot \nabla \lambda_{\Delta} d \mathbf{S}
$$

Recall that the secondary constraint is $\nabla \cdot\left(\rho_{0} \mathbf{u}\right)=\rho_{0} \nabla \cdot \mathbf{u}=0$. The solution of (5) is therefore

$$
\lambda_{\Delta}=\text { constant. }
$$


The second derivation concerns the step from (20b) to (24)). From (223), it follows that

$$
\frac{\delta \mathcal{F}[\Delta]}{\delta \mathbf{u}}=-\nabla \frac{\delta \mathcal{F}[\Delta]}{\delta \Delta} .
$$

Detailed analysis of (201) entails

$$
\begin{aligned}
& 0=\{\mathcal{F}[\Delta], \mathcal{H}\}+\int_{D} \lambda_{\rho}\left(\mathbf{x}^{\prime}\right)\left\{\mathcal{F}[\Delta], \rho\left(\mathbf{x}^{\prime}\right)\right\} d \mathbf{x}^{\prime} \\
&+\int_{D} \lambda_{\Delta}\left(\mathbf{x}^{\prime}\right)\left\{\mathcal{F}[\Delta], \Delta\left(\mathbf{x}^{\prime}\right)\right\} d \mathbf{x}^{\prime} \\
&=\int_{D} 2 \boldsymbol{\Omega} \times \mathbf{u} \cdot \nabla \frac{\delta \mathcal{F}}{\delta \Delta} d \mathbf{x}+\iint_{D, D^{\prime}} \lambda_{\rho}(\mathbf{x}) \nabla^{\prime} \cdot\left(\frac{\delta \mathcal{F}[\Delta]}{\delta \mathbf{u}\left(\mathbf{x}^{\prime}\right)}\right) \frac{\delta \rho(\mathbf{x})}{\delta \rho\left(\mathbf{x}^{\prime}\right)} d \mathbf{x} d \mathbf{x}^{\prime} \\
&+\iint_{D, D^{\prime}} \lambda_{\Delta}(\mathbf{x}) \frac{2 \Omega}{\rho_{0}} \times \frac{\delta \mathcal{F}[\Delta]}{\delta \mathbf{u}\left(\mathbf{x}^{\prime}\right)} \cdot \frac{\delta \nabla \cdot \mathbf{u}(\mathbf{x})}{\delta \mathbf{u}\left(\mathbf{x}^{\prime}\right)} d \mathbf{x} d \mathbf{x}^{\prime} .
\end{aligned}
$$

The third term in (可) can be shown to be zero as follows

$$
\begin{aligned}
& \iint_{D, D^{\prime}} \lambda_{\Delta}(\mathbf{x}) \frac{2 \boldsymbol{\Omega}}{\rho_{0}} \times \frac{\delta \mathcal{F}[\Delta]}{\delta \mathbf{u}\left(\mathbf{x}^{\prime}\right)} \cdot \frac{\delta \nabla \cdot \mathbf{u}(\mathbf{x})}{\delta \mathbf{u}\left(\mathbf{x}^{\prime}\right)} d \mathbf{x} d \mathbf{x}^{\prime} \\
& =-\iint_{D, D^{\prime}} \nabla \lambda_{\Delta}(\mathbf{x}) \cdot \frac{2 \boldsymbol{\Omega}}{\rho_{0}} \times \frac{\delta \mathcal{F}[\Delta]}{\delta \mathbf{u}\left(\mathbf{x}^{\prime}\right)} \cdot \frac{\delta \mathbf{u}(\mathbf{x})}{\delta \mathbf{u}\left(\mathbf{x}^{\prime}\right)} d \mathbf{x} d \mathbf{x}^{\prime} \\
& \left.+\int_{D^{\prime}} \int_{\partial D} \lambda_{\Delta}(\mathbf{x}) \frac{2 \boldsymbol{\Omega}}{\rho_{0}} \times \frac{\delta \mathcal{F}[\Delta]}{\delta \mathbf{u}\left(\mathbf{x}^{\prime}\right.}\right) \cdot \frac{\delta(\hat{\mathbf{n}} \cdot \mathbf{u}(\mathbf{x}))}{\delta \mathbf{u}\left(\mathbf{x}^{\prime}\right)} d S d \mathbf{x}^{\prime} \\
& =0
\end{aligned}
$$

since $\lambda_{\Delta}=$ cst (cf. ([G)) and by using ([4).

The second term in (ㅁ) can be reworked as follows

$$
\begin{aligned}
& \iint_{D, D^{\prime}} \lambda_{\rho}(\mathbf{x}) \nabla^{\prime} \cdot\left(\frac{\delta \mathcal{F}[\Delta]}{\delta \mathbf{u}\left(\mathbf{x}^{\prime}\right)}\right) \frac{\delta \rho(\mathbf{x})}{\delta \rho\left(\mathbf{x}^{\prime}\right)} d \mathbf{x} d \mathbf{x}^{\prime} \\
& =\int_{D} \lambda_{\rho} \nabla \cdot\left(\frac{\delta \mathcal{F}[\Delta]}{\delta \mathbf{u}}\right) d \mathbf{x} \\
& =\int_{D} \nabla \lambda_{\rho} \cdot \nabla\left(\frac{\delta \mathcal{F}[\Delta]}{\delta \Delta}\right) d \mathbf{x}-\int_{\partial D} \lambda_{\rho} \hat{\mathbf{n}} \cdot \nabla\left(\frac{\delta \mathcal{F}[\Delta]}{\delta \Delta}\right) d S \\
& =-\int_{D} \nabla^{2} \lambda_{\rho} \cdot \frac{\delta \mathcal{F}[\Delta]}{\delta \Delta} d \mathbf{x}+\int_{\partial D} \nabla \lambda_{\rho} \cdot \hat{\mathbf{n}} \frac{\delta \mathcal{F}[\Delta]}{\delta \Delta} d S .
\end{aligned}
$$


The second last boundary term $\hat{\mathbf{n}} \cdot \nabla(\delta \mathcal{F} / \delta \Delta)$ in ([D]) is zero because it is imposed as extra gauge or boundary condition, cf. an earlier remark.

When we combine $(\mathbb{D})$ and $(\mathbb{G})$, the final result ([24) in the main text is reached

$$
0=-\int_{D} \frac{\delta \mathcal{F}}{\delta \Delta}\left(\nabla^{2} \lambda_{\rho}+\nabla \cdot(2 \boldsymbol{\Omega} \times \mathbf{u})\right) d \mathbf{x}+\int_{\partial D} \frac{\delta \mathcal{F}}{\delta \Delta} \hat{\mathbf{n}} \cdot\left(\nabla \lambda_{\rho}+2 \boldsymbol{\Omega} \times \mathbf{u}\right) d S
$$

\section{Acknowledgments}

We would like to thank L.R.M. Maas for valuable discussions, suggestions and support. Furthermore, we acknowledge financial support of Technology Foundation STW for the project "A numerical wave tank for complex wave and current interactions".

\section{References}

[1] V.R. Ambati, O. Bokhove, Space-time discontinuous Galerkin discretization of rotating shallow water equations. J. Comp. Phys. 225 (2007) $1233-1261$.

[2] K.D. Aldridge, L.I. Lumb, Inertial waves identified in the earth's fluid core, Nature 325,(1987) 421-423.

[3] K.D. Aldridge, L.I. Lumb, G.A. Henderson, A Poincaré model for the earth's fluid core, Geophys. Astrophys.

[4] I. Anderson, A. Huyer, R.L. Smith, Near-inertial motions off the Oregon coast, J. Geophys. Res. 88 (1983) 5960-5972.

[5] V.I. Arnold, The Hamiltonian nature of the Euler equations in the dynamics of a rigid body and of an ideal fluid, Usp. Mat. Nauk. 24 (1966b) $225-226$.

[6] D.N. Arnold, F. Brezzi, B. Cockburn, L.D. Marini, Unified analysis of discontinuous Galerkin methods for elliptic problems, SIAM J. Numer. Anal. 39(5) (2002) 1749-1779. 
[7] O. Bokhove, On balanced models in Geophysical Fluid Dynamics: Hamiltonian formulation, constraints and formal stability, Large-Scale Atmosphere-Ocean Dynamics: Vol II: Geometric Methods and Models. Ed.J. Norbury and I. Roulstone, Cambridge University Press, Cambridge, (2002) 1-63.

[8] O. Bokhove, M. Oliver, Parcel Eulerian-Lagrangian fluid dynamics for rotating geophysical flows. Proc. Roy. Soc. A. 462 (2006) 2575-2592.

[9] B. Dintrans, M. Rieutord, L. Valdettaro, Gravito-inertial waves in a rotating stratified sphere or spherical shell. J. Fluid Mech. 398 (1999) 271-297.

[10] P. Dirac, Generalized Hamiltonian dynamics, Proc. R. Soc. Lond., Ser. A 246 (1958) 326-332.

[11] P.D. Fricker, H.M. Nepf, Bathymetry, stratification, and internal seiche structure. J. Geophys. Res. 105 (2000) 14237-14251.

[12] L.L. Fu, Observations and models of inertial waves in the deep ocean, Rev. Geophys. Space Phys. 19 (1981) 141-170.

[13] F.R. Gantmacher, Matrix Theory Vol.1, Chelsea, New York (1959).

[14] I.M. Gelfand, Lectures on Linear Algebra, Dover Publications (1989).

[15] T. Gelhard, G. Lube, M.A. Olshanskii, J.H. Starcke, Stabilized finite element schemes with LBB-stable elements for incompressible flows, J. Comput. Appl. Math. 177 (2005) 243-267.

[16] G.H Golub, C.F. van Loan, Matrix Computations, The Johns Hopkins University Press, Baltimore (1996).

[17] H. Greenspan, The Theory of Rotating Fluids. Cambridge University Press, Cambridge (1968).

[18] E. Hairer, C. Lubich, G. Wanner, Geometric Numerical Integration, Springer, Berlin (2006) 644 pp.

[19] H. van Haren, C. Millot, Rectilinear and circular inertial motions in the Western Mediterranean Sea, Oceanol. Acta, 51 (11) (2004) 1441-1455. 
[20] B. Leimkuhler, S. Reich, Simulating Hamiltonian Dynamics, Cambridge Monographs (2005) 379 pp.

[21] L.R.M. Maas, Wave focusing and ensuing mean flow due to symmetry breaking in rotating fluids, J. Fluid Mech. 437 (2001) 13-28.

[22] L.R.M. Maas, On the amphidromic structure of inertial waves in a rectangular parallelepiped, Fluid Dyn. Res. 33 (2003) 373-401.

[23] W.V.R. Malkus, Precession of the Earth as the cause of geomagnetism, Science 160 (1968), 259-264.

[24] R. Manasseh, Visualization of the flows in precessing tanks with internal baffles, Am. Inst. Aeronaut. Astronaut. J. 31 (1993) 312-318.

[25] A.M.M. Manders, L.R.M. Maas, Observations of inertial waves in a rectangular basin with one sloping boundary, J. Fluid Mech. 493 (2003) 59-88.

[26] J.E. Marsden, T.S. Ratiu, Introduction to Mechanics and Symmetry, Springer, second ed., New York, TAM 17 (1999).

[27] G. Matthies, G. Lube, On streamline-diffusion methods of inf-sup stable discretisations of the generalised Oseen problem, Preprint 2007-02, Institut für Numerische und Angewandte Mathematik, Georg-AugustUniversität Göttingen (2007).

[28] P.J. Morrison, Hamiltonian description of the ideal fluid, Rev. Mod. Phys. 70 (1998) 467-521.

[29] P.J. Morrison, J. M. Greene, Noncanonical Hamiltonian density formulation of hydrodynamics and ideal magnetohydrodynamics, Phys. Rev. Lett. 45 (1980) 790-794.

[30] S. Nurijanyan, O. Bokhove, L.R.M. Maas, Inertial waves in a rectangular parallelepiped, Phys. of Fluids (submitted, eprints copy: http: //eprints.eemcs.utwente.nl/22540/).

[31] S. Nurijanyan, J.J.W. van der Vegt, O. Bokhove, Hamiltonian discontinuous Galerkin FEM for linear, rotating incompressible Euler equations: inertial waves, (eprints copy: http://eprints.eemcs.utwente. nl/21124/). 
[32] L. Pesch, A. Bell, W.E.H. Solie, V.R. Ambati, O. Bokhove and J.J.W. van der Vegt, hpGEM - A software framework for discontinuous Galerkin finite element Methods, ACM Transactions on Mathematical Software, 33(4) (2007).

[33] O.M. Phillips, Energy transfer in rotating fluids by reflection of inertial waves, Phys. Fluids 6 (1963) 513-520.

[34] M. Rieutord, Inertial modes in the liquid core of the Earth, Phys. Earth Planet Interiors 91 (1995) 41-46.

[35] M. Rieutord, L. Valdettaro, Inertial waves in a rotating spherical shell. J. Fluid Mech. 341(1997) 77-99.

[36] R. Salmon, Semigeostrophic theory as a Dirac-bracket projection, J. Fluid Mech. (1988) 345-358.

[37] R. Salmon, Hamiltonian fluid mechanics, Annual Rev. Fluid Mech. 20 (1988) 225-256.

[38] B. Satish, B. Kris, E. Victor, D.G. William, K. Dinesh, G.K. Matthew, McInnes C. Lois, B.F. Smith, Z. Hong, PETSc users manual, Argonne National Laboratory, NL-95/11 - Revision 2.1.5 (2004).

[39] B. Satish, B. Kris, D.G. William, K. Dinesh, G.K. Matthew, McInnes C. Lois, B.F. Smith, Z. Hong, PETSc Web page http://www-unix.mcs. anl.gov/petsc (2001).

[40] K. Stewartson, On trapped oscillations of a rotating fluid in a thin spherical shell, Tellus XXII (6) (1971) 506-510.

[41] K. Stewartson, On trapped oscillations of a rotating fluid in a thin spherical shell ii. Tellus XXIV (4) (1972) 283-286.

[42] B.R. Sutherland, Internal Gravity Waves, Cambridge University Press (2010).

[43] J. Vanneste, O. Bokhove, Dirac-bracket approach to nearly geostrophic Hamiltonian balanced models, Physica D 164 (3-4) (2002) 152-167. 
[44] J.J.W. Van der Vegt, F. Iszak, O. Bokhove, Error analysis of a continuous-discontinuous Galerkin finite element model for generalized 2D vorticity dynamics, Siam J. Num. Anal. 45 (2007) 1349-1369.

[45] Y. Xu, J.J.W. van der Vegt, O. Bokhove, Discontinuous Hamiltonian finite element method for linear hyperbolic systems, J. Sci. Comput. 35 (2008) 241-265. 